\title{
Lead Slowing-Down Spectrometry Time Spectral Analysis for Spent Fuel Assay: FY12 Status Report
}
J Kulisek
ER Siciliano
KK Anderson
GA Warren
AM Casella

September 2012 


\title{
DISCLAIMER
}

This report was prepared as an account of work sponsored by an agency of the United States Government. Neither the United States Government nor any agency thereof, nor Battelle Memorial Institute, nor any of their employees, makes any warranty, express or implied, or assumes any legal liability or responsibility for the accuracy, completeness, or usefulness of any information, apparatus, product, or process disclosed, or represents that its use would not infringe privately owned rights. Reference herein to any specific commercial product, process, or service by trade name, trademark, manufacturer, or otherwise does not necessarily constitute or imply its endorsement, recommendation, or favoring by the United States Government or any agency thereof, or Battelle Memorial Institute. The views and opinions of authors expressed herein do not necessarily state or reflect those of the United States Government or any agency thereof.

\author{
PACIFIC NORTHWEST NATIONAL LABORATORY \\ operated by \\ BATTELLE \\ for the \\ UNITED STATES DEPARTMENT OF ENERGY \\ under Contract DE-AC05-76RL01830
}

Printed in the United States of America

Available to DOE and DOE contractors from the Office of Scientific and Technical Information,

P.O. Box 62, Oak Ridge, TN 37831-0062;

ph: (865) 576-8401

fax: (865) 576-5728

email: reports@adonis.osti.gov

\author{
Available to the public from the National Technical Information Service \\ 5301 Shawnee Rd., Alexandria, VA 22312 \\ ph: (800) 553-NTIS (6847) \\ email: orders@ntis.gov <http://www.ntis.gov/about/form.aspx> \\ Online ordering: http://www.ntis.gov
}




\title{
Lead Slowing-Down Spectrometry Time Spectral Analysis for Spent Fuel Assay: FY12 Status Report
}

\author{
J Kulisek ER Siciliano \\ KK Anderson GA Warren \\ AM Casella
}

September 2012

Prepared for the U. S. DEPARTMENT OF ENERGY under Contract DE-AC05-76RL01830

Pacific Northwest National Laboratory

Richland, WA 99354 


\section{Acknowledgments}

The authors would like to acknowledge the Department of Energy Fuel Cycle Research and Development Program, Material Protection, Accounting, and Control Technology (MPACT) Campaign for their support in funding this work. 


\section{Executive Summary}

Developing a method for the accurate, direct, and independent assay of the fissile isotopes in bulk materials (such as used fuel) from next-generation domestic nuclear fuel cycles is a goal of the Office of Nuclear Energy, Fuel Cycle R\&D, Material Protection and Control Technology (MPACT) Campaign. To meet this goal, MPACT supports a multi-institutional collaboration, of which PNNL is a part, to study the feasibility of Lead Slowing Down Spectroscopy (LSDS). This technique is an active, nondestructive assay method that has the potential to provide independent, direct measurement of plutonium and uranium isotopic masses in used fuel with an uncertainty considerably lower than the approximately $10 \%$ typical of today's confirmatory methods. This document is a progress report for FY2012 PNNL analysis and algorithm development.

Progress made by PNNL in FY2012 continues to indicate the promise of LSDS analysis and algorithms as applied to used-fuel assemblies. PNNL further refined the semi-empirical model developed in FY2011 based on singular value decomposition to numerically account for the effects of self-shielding. The average uncertainty in the plutonium mass across the NGSI-64 fuel assemblies was shown to be less than $3 \%$ using only six calibration assemblies with a $2 \%$ uncertainty in the isotopic masses. When calibrated against the six NGSI-64 fuel assemblies, the algorithm was able to determine the total plutonium mass within $<2 \%$ uncertainty for the 27 diversion cases also developed under NGSI. Two purely empirical algorithms were developed that do not require the use of plutonium isotopic fission chambers. The semiempirical and purely empirical algorithms were successfully tested using MCNPX simulations as well as applied to experimental data measured by Rensselaer Polytechnic Institute (RPI) using their LSDS. The algorithms were able to describe the ${ }^{235} \mathrm{U}$ masses of the RPI measurements with a root mean square error of $2.3 \%$. Analyses were conducted that provided valuable insight with regard to design requirements (e.g., lead stack size, neutron source location, sensitivity to ${ }^{235} \mathrm{U}$ in ${ }^{238} \mathrm{U}$ fission chambers) of an LSDS for the purpose of assaying used-fuel assemblies. Sensitivity studies were conducted that provide insight into ways the LSDS instrument can be improved by making it more sensitive to the center of the fuel assemblies.

In FY2013, PNNL will continue efforts to develop and refine design requirements of an LSDS for the ultimate purpose of assaying used-fuel assemblies. Future efforts will be directed toward more extensive experimental benchmarking of currently implemented time-spectra analysis algorithms. 


\title{
Symbols, Acronyms and/or Initialisms
}

BU
CT
GWd/MTU
HEU
IAEA
IE
ISU
LINAC
LSDS
MLE
LANL
MPACT
NDA
NGSI
PNNL
PWR
RMSE
RPI

\author{
Burn-up \\ Cooling time \\ Gigawatt days per metric ton of uranium \\ Highly enriched uranium \\ International Atomic Energy Agency \\ Initial enrichment \\ Idaho State University \\ Linear Accelerator \\ Lead Slowing-down spectroscopy \\ Maximum-likelihood estimation \\ Los Alamos National Laboratory \\ Material Protection and Control Technology \\ Non-destructive assay \\ Next Generation Safeguards Initiative \\ Pacific Northwest National Laboratory \\ Pressurized water reactor \\ Root mean square error \\ Rensselaer Polytechnic Institute
}




\section{Contents}

$\begin{array}{lll}1.0 & \text { Introduction } & 9\end{array}$

2.0 Principles of LSDS for Fuel Assay $\quad 11$

3.0 Time Spectral Analysis Algorithms $\quad 13$

$3.1 \quad$ Semi-Empirical Approach $\quad 14$

3.2 $\quad$ First Order Linear Model 15

3.3 Purely Empirical Second Order Model 16

4.0 Comparison of Results Obtained from the Various Time-Spectra Analysis Algorithms 18

4.1 Results for Simulations of Assay of Used-fuel assemblies $\quad 18$

4.2 Application of Algorithms to RPI's Experimental Data 21

5.0 LSDS and Semi-Empirical Algorithm Sensitivity Analyses 26

5.1 Sensitivity of the Semi-Empirical Algorithm to Uncertainties in Calibration Set Masses 26

5.2 Sensitivity to Number of Fuel Assemblies in Calibration Set 27

5.3 Sensitivity to Range of Neutron Slowing-Down Times Used in Analysis 28

5.4 Ability of LSDS for Full Volume Assay 29

5.5 Assays of Single and Nine Fresh Fuel Pin Arrays 30

5.6 Sensitivity to Water in Fuel Pins 31

6.0 Conceptual LSDS Design Studies $\quad 33$

6.1 Neutron Source Strength Requirements 33

6.2 Lead Size Requirements $\quad 34$

6.3 Level of ${ }^{235} \mathrm{U}$ impurities and cadmium $\quad 36$

$\begin{array}{lll}7.0 & \text { Future Work } & 39\end{array}$

$\begin{array}{lll}8.0 & \text { Conclusions } & 40\end{array}$

$\begin{array}{llr}9.0 & \text { References } & 41\end{array}$ 


\section{Figures}

Figure 2-1: Schematic of the PNNL model of a lead slowing-down spectrometer for fuel assay. 12

Figure 3-1: Example LSDS time spectra from the PNNL LSDS design. 13

Figure 4-1: Results for fissile plutonium mass estimates applying the semi-empirical algorithm on MCNPX-simulated assay of the fuel assemblies in the NGSI 64 version 1 library

Figure 4-2: Results for fissile plutonium mass estimates applying the semi-empirical algorithm on MCNPX-simulated assay of the 27 diversion assembly models

Figure 4-3: Results for ${ }^{235} \mathrm{U}$ mass estimates applying the semi-empirical algorithm on MCNPX-

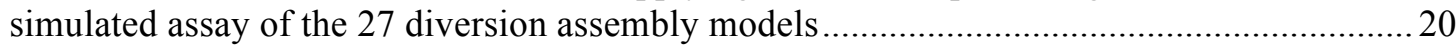

Figure 4-4: LSDS at the RPI Gaerttner Linear Accelerator.....................................................2 22

Figure 4-5: HEU discs wrapped in aluminum foil around the fresh SPERT fuel pin .................. 23

Figure 4-6: Measured ${ }^{238} \mathrm{U}$ assay signal and corresponding fit for the assay of a SPERT fuel pin,

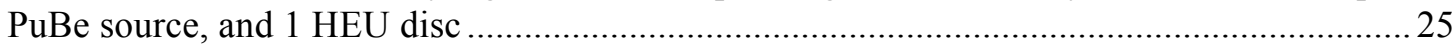

Figure 5-1: Results for ${ }^{235} \mathrm{U}$ and ${ }^{239} \mathrm{Pu}+{ }^{241} \mathrm{Pu}$ mass estimates applying the semi-empirical algorithm on MCNPX-simulated assay of the NGSI 64

Figure 5-2: Results for ${ }^{235} \mathrm{U}$ and ${ }^{239} \mathrm{Pu}+{ }^{241} \mathrm{Pu}$ mass estimates applying the semi-empirical algorithm on MCNPX-simulated assay of the 27 diversion assembly models

Figure 5-3: Results for ${ }^{235} \mathrm{U}$ and ${ }^{239} \mathrm{Pu}+{ }^{241} \mathrm{Pu}$ mass estimates for various calibration set sizes..... 28

Figure 5-4: Assay signal per total number of ${ }^{235} \mathrm{U},{ }^{239} \mathrm{Pu}$, and ${ }^{241} \mathrm{Pu}$ atoms as a function of depth from the inner to the outer perimeter of fuel pins of the assembly. Also shown is the interrogation neutron flux as a function of depth in the assembly

Figure 5-5: ${ }^{238} \mathrm{U}$ fission chamber signal obtained from the simulated assay of single fresh fuel pins of various wt\% of ${ }^{235} \mathrm{U}$ enrichments. The ${ }^{238} \mathrm{U}$ fission chamber signal signals are shown normalized to the signal for the $1 \mathrm{wt} \%$ enriched fuel pin.

Figure 5-6: ${ }^{238} \mathrm{U}$ fission chamber signal obtained from the simulated assay of nine fresh fuel pin arrays of various $w t \%$ of ${ }^{235} \mathrm{U}$ enrichments. The ${ }^{238} \mathrm{U}$ fission chamber signal signals are shown normalized to the signal for the $1 \mathrm{wt} \%$ enriched nine fuel pin array.

Figure 5-7: Simulated ${ }^{238} \mathrm{U}$ fission chamber signals for various wt $\%$ of water of each pin in the central row of fuel pins.

Figure 6-1: Number of source neutrons required in an LSDS to achieve the same level of fractional uncertainties in the MCNPX-simulated ${ }^{238} \mathrm{U}$ fission chamber signal

Figure 6-2: Simulated ${ }^{238} \mathrm{U}$ fission chamber signal for cylindrical LSDS instruments of various size (lead outer dimensions) 
Figure 6-3: Simulated ${ }^{238} \mathrm{U}$ fission chamber signal for cylindrical LSDS instruments of various size (lead outer dimensions) 36

Figure 6-4: Sensitivity to ${ }^{235} \mathrm{U}$ impurities in a ${ }^{238} \mathrm{U}$ fission chamber... 37 


\section{Tables}

Table 3-1: Calibration set of fuel assemblies used in semi-empirical algorithm

Table 4-1: Average RMSE values for total fissile plutonium and ${ }^{235} \mathrm{U}$ mass for simulated assays 21

Table 4-2: Calibration set for first and second order empirical algorithms for experimental data. 23

Table 4-3: Relative errors for ${ }^{235} \mathrm{U}$ mass estimates for the first and second order empirical algoirthms

Table 4-4: Calibration set for the semi-empirical algorithm for experimental data. 24

Table 4-5: Relative errors for ${ }^{235} \mathrm{U}$ mass estimates using the semi-empirical algorithm. .24

Table 5-1: Average RMSE values obtained for ${ }^{235} \mathrm{U}$ and ${ }^{239} \mathrm{Pu}+{ }^{241} \mathrm{Pu}$ masses in the NGSI 64 using various slowing-down time ranges in the semi-empirical algorithm .............................2 29 


\subsection{Introduction}

Nondestructive assay (NDA) techniques for determining the quantity of plutonium isotopes (i.e., ${ }^{239} \mathrm{Pu}$, ${ }^{240} \mathrm{Pu},{ }^{241} \mathrm{Pu}$ ) in used-fuel assemblies is of great interest for nuclear safeguards operations with respect to fissile material accountability, determining shipper-receiver differences, and maintaining continuity of knowledge. Such NDA techniques can also be used to support criticality calculations by verifying fuel burnup in order to maximize used-fuel capacity in fuel storage, and to optimize the efficient transport of used fuel.

Current NDA methods infer total plutonium mass using a combination of burnup codes for calculating isotopic inventories and passive measurements of easily measured isotopes in used fuel (e.g., ${ }^{137} \mathrm{Cs}$ and ${ }^{244} \mathrm{Cm}$ ). The International Atomic Energy Agency (IAEA) has determined that such methods typically carry a plutonium uncertainty of approximately $10 \%[1]$.

To address these issues, this project is studying the application of lead slowing down spectrometry (LSDS) for measuring fissile isotopic masses in used-fuel assemblies. LSDS is a well-established active interrogation technique that has been used for nuclear cross-section measurements for several decades $[2,3]$. The goal of this effort is to use LSDS to directly measure fissile isotopes (e.g., ${ }^{235} \mathrm{U},{ }^{239} \mathrm{Pu}$ and ${ }^{241} \mathrm{Pu}$ ) in used-fuel assemblies with significantly better accuracy than current methods, with minimal externally provided (operator-declared) information, and in a time-efficient manner. Primary target applications for the LSDS are at the receiving end of a reprocessing/pyro-processing, waste processing, or fuel storage facility to establish the initial accountancy of plutonium mass through direct measurement.

Earlier work by PNNL explored the use of neural networks capable of "learning" the effects of selfshielding and compensating for nonlinearities between signal strength and isotopic masses [4]. However, that work did not address the objective of truly direct measurement and required extensive calibration using well-known spent-fuel assembly standards - an expensive and in some cases, impossible, requirement. Following this, the authors explored the use of a nonlinear correction to the linear relationship using cubic splines to approximate the self-shielding effect over all slowing times [5]. The need for analyst intervention in defining knot points, and the limitations of the splines methodology to accurately track the sharp features of self-shielding, were considerable downsides to the splines approach. Subsequently, an analytical model for self-shielding was developed that relied on tabulated nuclear data but left the mass of fissile and attenuating isotopes as fitting parameters [6]. The method yielded fairly reasonable total plutonium mass errors within 5\% over a narrow fuel parameter range and showed that LSDS was capable of full-volume assay; however, the method yielded $\sim 10 \%$ error on total plutonium mass [7] over the entire parameter range spanned by the 64 assembly models in version 1 of the next generation safeguards initiative used fuel assembly library [8], as modeled in MCNPX 2.6.0 [9]. Therefore, in FY2011, in addition to continuing to study and refine its analytical model, PNNL developed a semi-empirical algorithm, which showed promise of significantly reducing the uncertainties of the extracted isotopic masses to below 3\% over the entire parameter range spanned by the NGSI 64 used fuel assembly library [10].

Significant progress toward this goal was made in FY2012. PNNL demonstrated, using the semiempirical algorithm developed in FY2011, the potential of the LSDS to determine the total fissile plutonium mass within an average of 3\% over the entire NGSI 64 library and the associated set of 27 diversion assemblies using a small calibration set consisting of only 6 used-fuel assemblies. This was a significant reduction from the initial calibration set consisting of the entire NGSI 64 library used in 
FY2011. This empirical algorithm was also successfully applied to experimental data measured by RPI in FY2012 to estimate ${ }^{235} \mathrm{U}$ masses in fresh fuel and highly enriched uranium (HEU) discs. Further, PNNL investigated the sensitivity of the empirical algorithm to uncertainties in the calibration set masses and the number of fuel assemblies used in the calibration set. The ability of the LSDS to measure the fissile material in the inner rows of the fuel assemblies was also examined. PNNL additionally developed a purely empirical algorithm that could be implemented using only threshold fission chambers, lined with either ${ }^{238} \mathrm{U}$ or ${ }^{232} \mathrm{Th}$, since the original empirical algorithm (developed in FY2011) relies on signals measured by isotopic fission chambers containing material that may be difficult to acquire (i.e., ${ }^{235} \mathrm{U}$, ${ }^{239} \mathrm{Pu}$, but particularly ${ }^{241} \mathrm{Pu}$ ).

PNNL also studied aspects related to the conceptual design of an LSDS-based system. Issues such as neutron source strength requirements, the size of the lead stack and the possible use of cadmium to reduce the influence of ${ }^{235} \mathrm{U}$ in the ${ }^{238} \mathrm{U}$ fission chambers were examined. 


\subsection{Principles of LSDS for Fuel Assay}

The use of LSDS for the NDA of used-fuel assemblies is based on the unique resonance structure of the cross sections of the isotopes in the fuel. These resonances are strong functions of the incident neutron energy. A simplified schematic of the PNNL design of a spectrometer for use in the NDA of used nuclear fuel is shown in Figure 2-1. This figure also illustrates the process for determining the isotopic masses in the fuel assembly. The interrogating neutrons are injected into the lead by a pulsed neutron source (e.g., linear accelerator (LINAC)), initially having several MeV of energy. Within approximately a microsecond, they lose energy via inelastic collisions with the lead nuclei such that the mean interrogating neutron energy decreases to approximately $100 \mathrm{keV}$. At that time (and neutron energy), elastic scattering becomes the dominant interaction process. Then the relationship between neutron energy $E$ and slowing time $t$, and the energy resolution, are given by [11]

$$
\begin{aligned}
& E=\frac{k}{\left(t+t_{0}\right)^{2}}, \\
& \frac{\Delta E}{E}=\sqrt{\frac{8}{3 A}},
\end{aligned}
$$

where $k$ and $t_{0}$ are parameters of the spectrometer, and $A$ is the atomic mass of the material of the spectrometer. Note that the resolution is independent of the time in this approximation. An underlying assumption of these equations is that the lead is completely free from impurities, particularly those having light atomic mass, such as hydrogen. The presence of the fuel assembly in the spectrometer interrogation chamber also has an appreciable effect on the energy as a function of slowing time and the energy resolution [6].

As the interrogating neutrons slow down in the lead, they induce fissions in the various isotopes of the sample. The prompt fission neutrons emitted by these fissile isotopes contribute to the signal generated in the assay-signal sensors (e.g., fission chambers containing ${ }^{232} \mathrm{Th}$ or ${ }^{238} \mathrm{U}$ ). The fission neutrons can be distinguished from the interrogating neutrons by their energy. $A s^{232} \mathrm{Th}$ and ${ }^{238} \mathrm{U}$ have very low fission cross sections $(<100 \mu \mathrm{b})$ below $100 \mathrm{keV}$, they make ideal materials for assay chambers to detect the fast fission neutrons. 


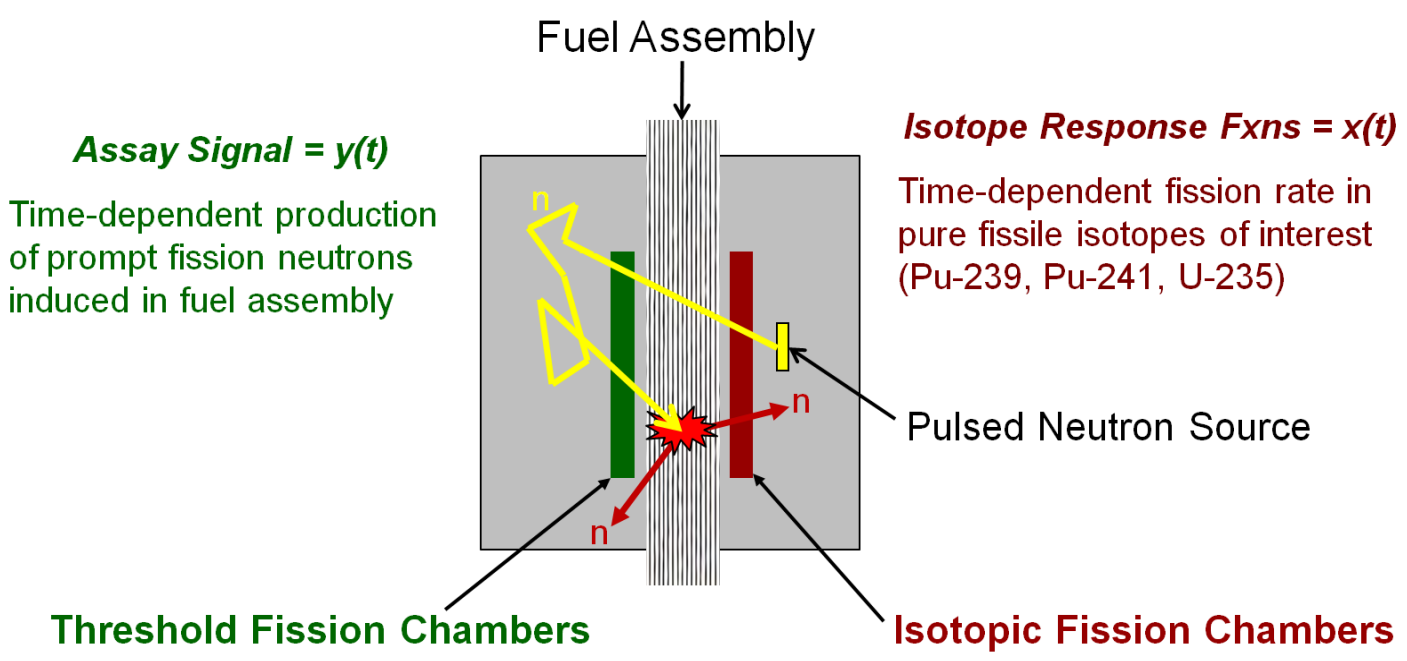

Figure 2-1: Schematic of the PNNL model of a lead slowing-down spectrometer for fuel assay. The interrogating neutron population induces fission in the fuel assembly and isotopic fission chambers, while the prompt fission neutrons generate a signal in the threshold or assay fission chambers.

Currently, no spectrometer is capable of handling used-fuel assemblies in order to evaluate LSDS methods described in this report. Therefore, this work currently relies on a modeling library (version 1) of 64 fuel assemblies developed by LANL [8] for the Next Generation Safeguard Initiative (NGSI). This collection of modeled fuel assemblies will be referred to as the NGSI 64. The assemblies range in initial ${ }^{235} \mathrm{U}$ loading from $2 \%$ to $5 \%$, in burnup from 15 to $60 \mathrm{GWd} / \mathrm{MTU}$, and in cooling time from 1 to 80 years. The hydrogen concentration in the fuel cladding of each assembly is set as $10 \mathrm{ppm}$ per $1 \mathrm{GWd} / \mathrm{MTU}$. Variation in radial burnup within each fuel pin, as well as variation in burnup among each separate fuel pin are included within each of the 64 fuel assembly models. In addition, the NGSI library consists of 27 diversion assemblies, which were modified from the fuel assemblies in the first set of 64 (which had an initial enrichment of $4 \mathrm{wt} \%{ }^{235} \mathrm{U}$, a cooling time of 5 years, and burnups of 15,30 , and $45 \mathrm{GWd} / \mathrm{MTU}$ ). These assemblies were modified to form the diversion assemblies by removing various numbers $(8,24$, and 40) of fuel pins from the inner, middle, and outer regions of the assemblies and replacing them with fuel pins containing depleted uranium $\left(0.2 \mathrm{wt} \%{ }^{235} \mathrm{U}\right)$ dioxide. In MCNPX, the NGSI 64 fuel assembly models and 27 diversion assembly models were placed inside the assay chamber of a notational spectrometer described in [6] to simulate the assay and to evaluate the mathematical models used to extract the masses of the isotopes. 


\subsection{Time Spectral Analysis Algorithms}

In the PNNL approach, isotopic fission chambers measure the isotopic response to the neutron field inside the lead stack. The isotopic fission chambers are comprised of one of the isotopes of interest (e.g., ${ }^{235} \mathrm{U}$ and ${ }^{239} \mathrm{Pu}$ ) assumed to be present in the fuel assembly. The individual signals generated from induced fission in these chambers, $x_{i}(t)$, serve to deconvolve the assay signal, $y(t)$, which is generated by the threshold fission chambers (lined with either ${ }^{238} \mathrm{U}$ or ${ }^{232} \mathrm{Th}$ ). The threshold fission chambers are sensitive to the prompt fission neutrons emitted from the fuel. Examples of simulated isotope response functions and assay signals are shown in Figure 3-1.
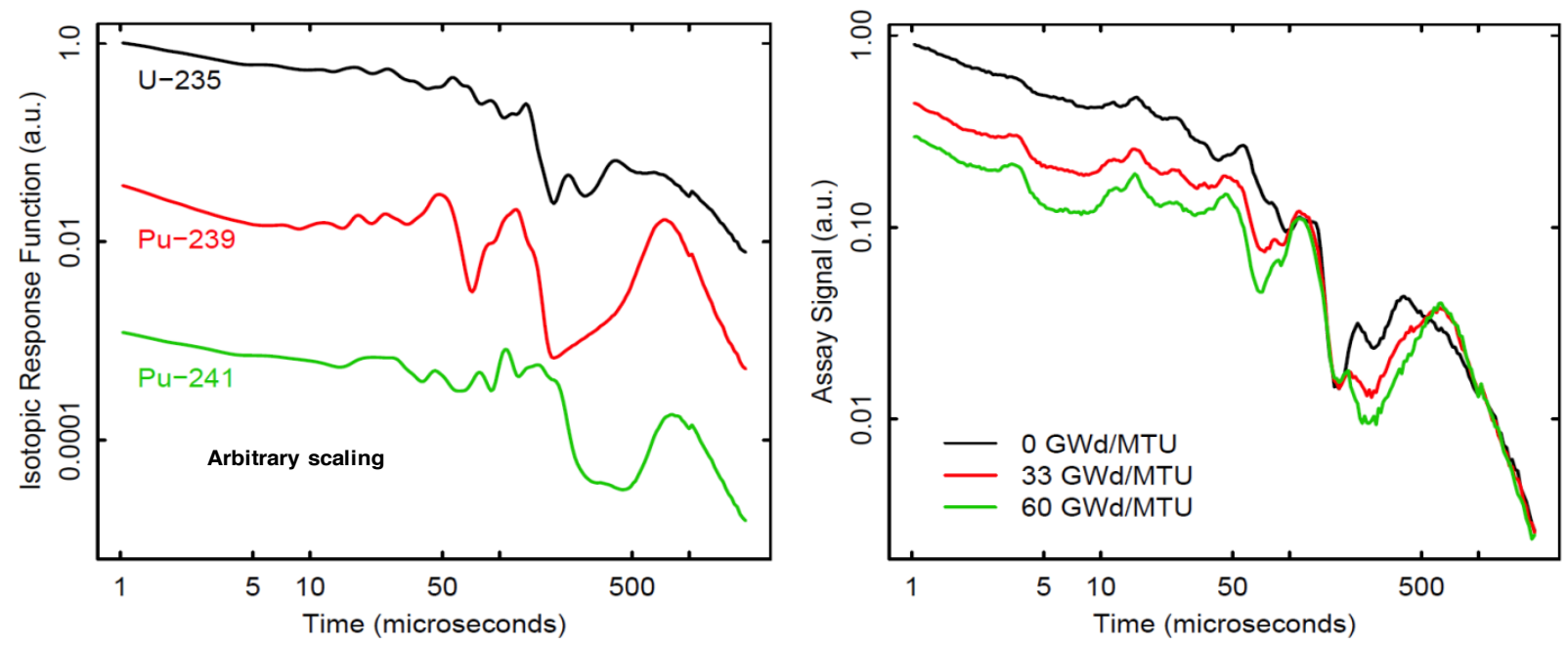

Figure 3-1: Example LSDS time spectra from the PNNL LSDS design: Isotope response functions, $x(t)$, for the three primary fissile isotopes of interest (left), and simulated assay signals, $y(t)$, from PWR fuels of various burnup levels (right) [6].

Ideally, if self-shielding were insignificant, we would expect the assay signal $y(t)$ to be a linear combination of the isotopic response functions, $x_{i}(t)$,

$$
y(t)=C\left[a_{235} x_{235}(t)+a_{239} x_{239}(t)+a_{241} x_{241}(t)\right]
$$

where 235, 239 and 241 indicate ${ }^{235} \mathrm{U},{ }^{239} \mathrm{Pu}$ and ${ }^{241} \mathrm{Pu}$, respectively. In (3), it is assumed for simplicity that the efficiencies the isotopic fission chambers are the same. The isotopic coefficients, $a_{i}$, are proportional to the corresponding isotopic masses, $m_{i}$, in the fuel. The constant $C$ is used to account for the different efficiencies of the threshold-reaction assay chambers, and the isotopic response chambers.

Realistically, the self-shielding effect caused by the presence of strong absorbers in the fuel assembly is significant and must be taken into account. This self-shielding effect modifies the interrogating neutron flux such that volumetric average flux over the fuel assembly, $\bar{\varphi}_{\text {fuel }}(E, t)$, is not equal to the flux in the fission chambers, $\varphi_{\text {detectors }}(E, t)$. In order to account for the self-shielding, a time-dependent selfshielding function, $f(t)$, is introduced: 


$$
y(t)=f(t) C \sum_{i} \frac{m_{i} v_{i}}{A_{i}} x_{i}(t)
$$

where $i$ indicates the fissile isotope, $v_{i}$ is the average number of neutrons released per fission, $A_{i}$ is the atomic mass, and $m_{i}$ is the mass of the isotope $i$. Equation (4) assumes each isotopic fission chamber contains the same number of fissile nuclei in addition to the assumptions upon which (3) is based. The self-shielding function, $f(t)$, is defined as

$$
f(t)=\frac{\bar{\varphi}_{\text {fuel }}(E, t)}{\varphi_{\text {detectors }}(E, t)} .
$$

Note that there is no energy-dependence in $f(t)$ in its definition in (5). Therefore, (4) and (5) assume that $\phi_{\text {detectors }}(E, t)$ and $\bar{\phi}_{\text {fuel }}(E, t)$ have the same energy spectral shape at any instant in time over the range of slowing-down times used in the analysis. In this scheme, in order to extract the isotopic masses $m_{i}$, it is necessary to measure the assay signal $y(t)$ and the isotopic responses $x_{i}(t)$ as well as have a means to determine the self-shielding function $f(t)$.

\subsection{Semi-Empirical Approach}

In FY2011, a semi-empirical model was developed to improve upon the analytical approach discussed in [10]. This approach was based on calibration and a numerical approximation to $f(t)$ using singular value decomposition (SVD) [12]. The SVD technique reduced the data to a set of empirical basis vectors to approximate $f(t)$ 's for each of the 64 NGSI fuels [13].

For the calibration step of the algorithm, six of the NGSI 64 fuel assemblies were chosen as a calibration set. It was initially assumed that true masses of the fissile isotopes were perfectly known. These six calibration fuel assemblies are described in Table 3-1, along with their fissile isotopic masses contained in the modeled one-meter-long section of the fuel assembly. The acronyms BU, IE, and CT stand for burnup, initial enrichment, and cooling time, respectively. Larger calibration sets were also explored, but it was found that a calibration set of six was the minimum set to accurately extract the isotopic masses.

Table 3-1: Calibration set of fuel assemblies used in semi-empirical algorithm

\begin{tabular}{|c|c|c|c|}
\hline \multirow{2}{*}{$\begin{array}{c}\text { Fuel Assembly Description } \\
\text { (BU, IE, CT) }\end{array}$} & \multicolumn{3}{|c|}{ Masses (g) } \\
\cline { 2 - 4 } & ${ }^{\mathbf{2 3 5} \mathbf{U}}$ & ${ }^{\mathbf{2 3 9}} \mathbf{P u}$ & ${ }^{\mathbf{2 4 1}} \mathbf{P u}$ \\
\hline $15 \mathrm{GWd} / \mathrm{MTU}, 2 \mathrm{wt} \%, 1 \mathrm{yr}$ & 1125 & 554 & 82 \\
\hline $30 \mathrm{GWd} / \mathrm{MTU}, 2 \mathrm{wt} \%, 1 \mathrm{yr}$ & 472 & 643 & 167 \\
\hline $30 \mathrm{GWd} / \mathrm{MTU}, 5 \mathrm{wt} \%, 20 \mathrm{yr}$ & 2933 & 768 & 53 \\
\hline $45 \mathrm{GWd} / \mathrm{MTU}, 2 \mathrm{wt} \%, 1 \mathrm{yr}$ & 186 & 668 & 208 \\
\hline $45 \mathrm{GWd} / \mathrm{MTU}, 5 \mathrm{wt} \%, 20 \mathrm{yr}$ & 1813 & 828 & 82 \\
\hline $60 \mathrm{GWd} / \mathrm{MTU}, 5 \mathrm{wt} \%, 20 \mathrm{yr}$ & 1059 & 854 & 106 \\
\hline
\end{tabular}

The quantities of $Y(t)$ and $X_{i}(t)$ are observables, obtained by scaling the raw MCNP tally results, $y(t)$ and $x_{i}(t)$, assuming $10^{16}$ total neutrons from the pulsed neutron source and absolute detector efficiencies for 
the modeled fission chamber design of a cylinder of $60 \mathrm{~cm}$ in height by $1.645 \mathrm{~cm}$ in radius, lined with an active layer of $1 \mathrm{mg} / \mathrm{cm}^{2}$. Poisson noise reflected the simulated statistics in each bin was then added to these observables. For each of the six calibration set assemblies given in Table 3-1, the values of $Y(t)$ and $X_{i}(t)$, were substituted for $y(t)$ and $x_{i}(t)$, respectively, in (4), and (4) was solved to obtain $f(t)$. The resulting six $f(t)$ 's, with a value of 1.0 subtracted to ensure mathematical non-degeneracy, were used to form $A$, a $6 \times 162$ matrix, where the 162 columns represent the 162 logarithmically-spaced time bins used in the MCNPX simulations, ranging from $20 \mu$ s to $2000 \mu \mathrm{s}$.

The SVD of $A$ was calculated, factoring $A$ into three matrices: unitary matrix $U$, diagonal matrix $S$, and matrix $V^{T}$,

$$
A_{6 \times 162}=U_{6 \times 6} S_{6 \times 6} V_{6 \times 162}^{T}
$$

The columns of $V^{T}$ form an orthogonal set of basis vectors, $B_{k}$, that span the column space of $A^{T}$, such that any of the six $f(t)$ 's, with a value of 1.0 subtracted (columns of $A^{T}$ ), can be represented by a linear combination of these six basis vectors. Five of the six basis vectors in $V^{T}$ were used to numerically approximate $f(t)$

$$
f(t) \approx 1.0+\sum_{k=1}^{5} b_{k} B_{k}(t),
$$

where the $b_{k}$ 's are left as unknown constants to be fit. It has been determined that using more than five basis vectors does not significantly reduce the errors in calculating the fissile isotopic masses [16]. For each fuel assembly assayed, (7) was substituted into (4), and the simulated isotopic fission chamber count rate, $X_{i}(t)$, was also substituted into (4) for $x_{i}(t)$, to form the calculated assay signal

$$
\hat{Y}(t)=\left[1.0+\sum_{k=1}^{5} b_{k} B_{k}(t)\right] C \sum_{i} \frac{m_{i} v_{i}}{A_{i}} X_{i}(t) .
$$

Nonlinear maximum likelihood estimation (MLE) (see Appendix A) was then used to solve for the masses, $m_{i}$, of the fissile isotopes and constants, $b_{k}$. In MLE, the fit is performed by minimizing the objective function

$$
R=\sum_{j=1}^{162}\left\{\hat{Y}\left(t_{j}\right)-Y\left(t_{j}\right) \times \log \left[\hat{Y}\left(t_{j}\right)\right]\right\},
$$

where the sum is performed over the 162 time bins used in the simulation. For this calculation, the calculated assay signal $\hat{Y}(t)$ from (8) and the true assay signal $Y(t)$ were substituted into (9).

\subsection{First Order Linear Model}

The semi-empirical model described in section 3.1 has demonstrated great potential for estimating the fissile isotopic masses. However, it is desirable to have an algorithm which does not rely on signals from the isotopic fission chambers, since these fission chambers require material, namely ${ }^{239} \mathrm{Pu}$ and especially ${ }^{241} \mathrm{Pu}$, which may be difficult to acquire (it should be mentioned, though, that isotopic fission chambers 
containing ${ }^{239} \mathrm{Pu}[14]$ as well as ${ }^{241} \mathrm{Pu}[15]$ exist and are currently being used). Therefore purely empirical first and second order models, based on Poisson regression, were developed. These models are also based on using a well-known calibration set of fuel assemblies but only rely upon the assay signal from threshold fission chambers, lined with either ${ }^{238} \mathrm{U}$ or ${ }^{232} \mathrm{Th}$. In Appendix B, it is shown that these isotopic fission chambers need not be $100 \%$ isotopically pure in order for the theory behind the PNNL model described by (4) to be valid. In the purely empirical approach, the basic concept is to develop a matrix that relates the masses of the calibration assemblies to their assay signals, and then to use that matrix relation to extract masses from assemblies with unknown masses.

\section{Calibration Procedure}

In the application of the linear empirical model, a set of $k$ used-fuel assemblies from NGSI 64 are chosen as calibrations. The MCNPX model of the LSDS currently assumes 162 time bins ranging in slowingdown times from approximately $20 \mu$ s to $2000 \mu$ s. Unlike the semi-empirical model discussed in section 3.1, the linear empirical model allows for any of the isotopic masses to be estimated (e.g., ${ }^{238} \mathrm{U}$ and ${ }^{240} \mathrm{Pu}$ ), not just the fissile isotopes. Plutonium-240 is of interest because it is included in the IAEA's definition of total plutonium. We denote the number of isotopic masses to be estimated as $p$. The assay fission chamber responses, $Y(t)$, from the $k$ calibration assemblies are used to form a matrix $Y_{162 \times k}=\left[Y_{1}(t) Y_{2}(t) \ldots Y_{k}(t)\right]$. The $p$ known isotopic masses of interest in the $k$ calibration assemblies are used to form a matrix $M_{p \times k}$. Equation (10) is then solved for matrix $B_{162 \times p}$ and error matrix $E_{162 \times k}$, using MLE (see Appendix A):

$$
Y_{162 \times k}=B_{162 \times p} M_{p \times k}+E_{162 \times k} .
$$

\section{Fitting Procedure}

In the fitting procedure, an assay signal, $Y(t)$ from a ${ }^{238} \mathrm{U}$ or ${ }^{232} \mathrm{Th}$ fission chamber is obtained from the assay of a used fuel assembly containing unknown amounts of the isotopes of interest. Using $Y(t)$ and matrix $B_{162 \times p}$ determined from the calibration procedure, the vector $m$ containing the $p$ isotopic masses of interest, are extracted by using MLE to solve

$$
Y(t)_{162 \times 1}=B_{162 \times p} m_{p \times 1}+e_{162 \times 1} .
$$

where $e$ is the error offset obtained from the fitted solution. The matrix and vector dimensions are included as subscripts in (11) for clarity.

\subsection{Purely Empirical Second Order Model}

Since the self-attenuation function is highly non-linear, a purely empirical model, second order with respect to the isotopic masses, was developed to more accurately estimate the isotopic masses from the used fuel assembly assays. One disadvantage of this approach is that the second order algorithm naturally requires more calibration assemblies, since $2^{\text {nd }}$ order mass terms are required in addition to $1^{\text {st }}$ order mass terms. 


\section{Calibration Procedure}

In the application of the $2^{\text {nd }}$ order empirical model, $k$ well-characterized calibration assemblies are chosen for the calibration set. As in the first-order empirical model, matrix $Y_{162 \times k}=\left[Y_{1}(t) Y_{2}(t) \ldots Y_{k}(t)\right]$ is formed from the fission chamber assay signals from the assay of the calibration assemblies. Matrix $M_{p \times k}$ contains the $p$ known isotopic masses of interest in the $k$ calibration fuel assemblies, and the elements in matrix $Q_{p \times k}$ are the square of each of these known isotopic masses. Cross terms in the masses (e.g., $m_{239} \times$ $m_{235}$ ) were not included in $Q_{p \times k}$, since the algorithm would have required too many fitting parameters and calibration assemblies. The $Y, M$ and $Q$ matrices are related by

$$
Y_{162 \times k}=C_{162 \times p} M_{p \times k}+D_{162 \times p} Q_{p \times k}+E_{162 \times k},
$$

where the matrices $C_{162 \times p}, D_{162 \times p}$, and $E_{162 \times k}$ are solved using MLE. Matrices $C_{162 \times p}$ and $D_{162 \times p}$ then contain the empirical basis vectors to be used for the assay of unknown fuel assemblies, and matrix $E_{162 \times k}$ is the error offset matrix resulting from the MLE.

\section{Fitting Procedure}

In the fitting procedure of the second order empirical model, an assay signal, $Y(t)$ from a ${ }^{238} \mathrm{U}$ or ${ }^{232} \mathrm{Th}$ fission chamber, is obtained from the assay of a used fuel assembly containing unknown amounts of the isotopes of interest. Using $Y(t)$ and matrices $C_{162 \times p}$ and $D_{162 \times p}$ determined from the calibration procedure, the vectors $m_{p \times 1}$ and $q_{p \times 1}$, which contain the $p$ unknown isotopic masses of interest and square of these isotopic masses, respectively, are extracted by using MLE to solve

$$
Y(t)_{162 \times 1}=C_{162 \times p} m_{p \times 1}+D_{162 \times p} q_{p \times 1}+e_{162 \times 1}
$$

where $e_{162 \times 1}$ is the error offset obtained from the fitted solution. The matrix and vector dimensions are included as subscripts for clarity.

Although this purely second-order model produced significantly better results than the purely empirical first-order model on average, the squared masses, contained in $q_{p \times 1}$, result in difficulties. For example, in addition to requiring more fuel assemblies in the calibration set, the squares of the masses result in large numbers (particularly for ${ }^{238} \mathrm{U}$ ) and numerical instabilities. 


\subsection{Comparison of Results Obtained from the Various Time- Spectra Analysis Algorithms}

The algorithms discussed in sections 3.1-3.3 were applied to both simulated assay data as well as measured data obtained from experiments conducted at RPI. In this section, we compare the extracted fissile isotopic masses estimated using the semi-empirical, purely empirical first order, and purely empirical second order algorithms for both types of data.

\subsection{Results for Simulations of Assay of Used-fuel assemblies}

Comparisons of the calculated masses from the semi-empirical method and the true masses for ${ }^{239} \mathrm{Pu}$ and

${ }^{241} \mathrm{Pu}$ are shown in Figure 4-1. The calculated masses were obtained by applying the semi-empirical method described in section 3.1 to the MCNPX-simulated assays of each of the used-fuel assemblies in the NGSI 64 version library. The indices shown on the independent axis of Figure 4-1, were assigned to the used-fuel assemblies such that each successive group of 16 consecutive indices correspond to fuels having equal burnup, groups of 4 consecutive indices having equal $\mathrm{wt} \%$ of ${ }^{235} \mathrm{U}$ initial enrichments, and every fourth index having equal cooling time. As shown in Figure 4-1, excellent agreement between the calculated and true plutonium masses were obtained over the entire NGSI 64 library.

The semi-empirical algorithm was also applied to the 27 diversion assembly models, and the corresponding results for plutonium mass estimates are shown in Figure 4-2. As shown, the calculated and true plutonium masses are in very good agreement for the 27 diversion assemblies as well. These results are promising, since the diversion assemblies are quite different from the calibration set assemblies taken directly from the NGSI 64. Also, it is interesting to note that the level of agreement between the mass estimates and the true masses of plutonium was independent of whether the rods were diverted from the inner, middle, or outer zones of the fuel assembly. The same could not be said of the calculated ${ }^{235} \mathrm{U}$ masses, for which better agreement was obtained for the cases for which the fuel rods were diverted from plutonium the outer zones of the assemblies, as indicated in Figure 4-3. Since there is significantly less ${ }^{239} \mathrm{Pu}$ and ${ }^{241} \mathrm{Pu}$ than ${ }^{235} \mathrm{U}$ in these diversion cases, these results suggest that statistical uncertainties are at least as significant of an impact as the geometric sensitivity in introducing error in the calculated plutonium masses. 


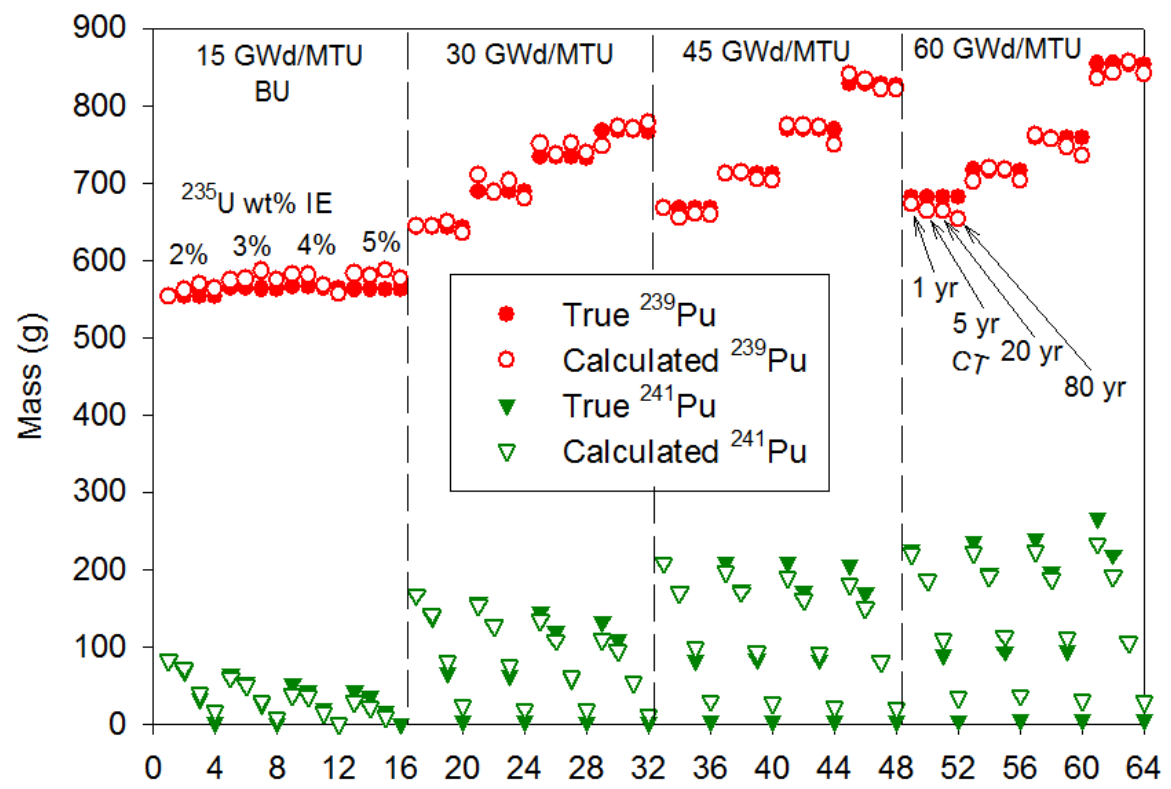

Fuel Index (\#)

Figure 4-1: Results for fissile plutonium mass estimates applying the semi-empirical algorithm on MCNPX-simulated assay of the fuel assemblies in the NGSI 64 version 1 library

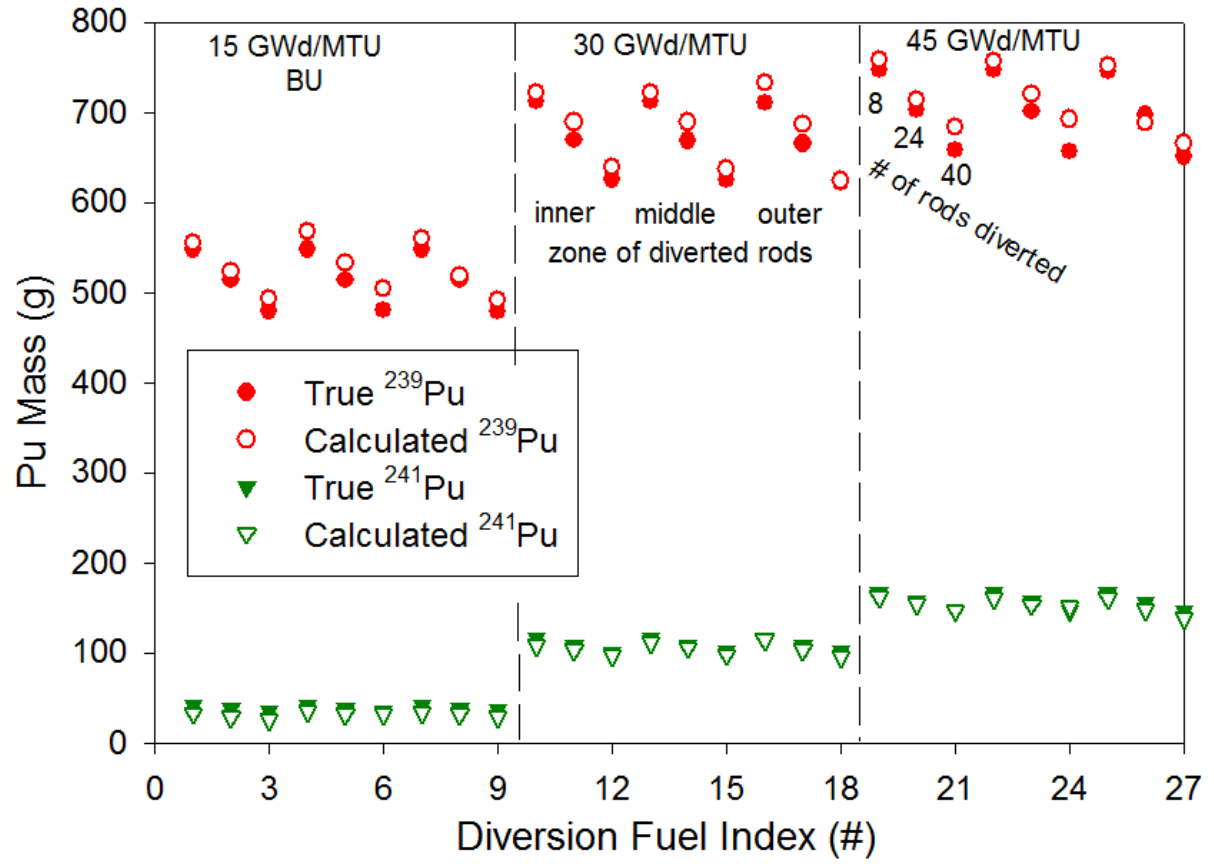

Figure 4-2: Results for fissile plutonium mass estimates applying the semi-empirical algorithm on MCNPX-simulated assay of the 27 diversion assembly models 


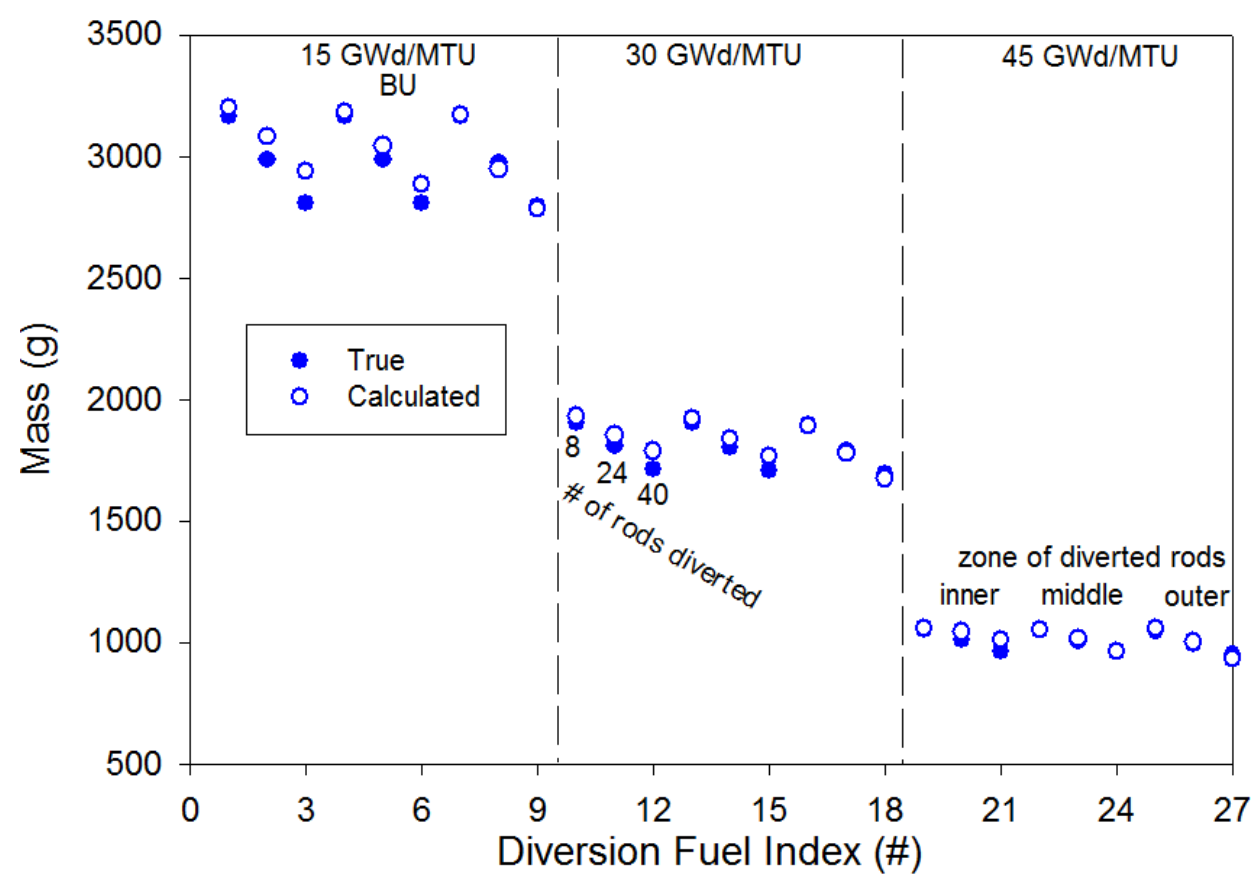

Figure 4-3: Results for ${ }^{235} \mathrm{U}$ mass estimates applying the semi-empirical algorithm on MCNPXsimulated assay of the 27 diversion assembly models

The average relative root-mean squared error,

$$
\operatorname{RMSE}(\%)=100 \times \sqrt{\frac{1}{N} \sum_{i}^{N}\left(\frac{\text { true }_{i}-\text { estimate }_{i}}{\text { true }_{i}}\right)^{2}},
$$

from several analyses for the total fissile plutonium $\left({ }^{239} \mathrm{Pu}+{ }^{241} \mathrm{Pu}\right.$ mass $)$ and the ${ }^{235} \mathrm{U}$ mass over the entire NGSI 64 library (excluding the 6-assembly calibration set) are given in Table 4-1. The first row contains the results for the semi-empirical algorithm. Results for the diversion assemblies are better than for the NGSI 64, likely due to the fact that the diversion assemblies did not contain the extreme cases in the NGSI 64 (e.g., $60 \mathrm{GWd} / \mathrm{MTU}$ BU, $2 \mathrm{wt} \%$ IE, $80 \mathrm{yr}$ CT). Also shown in Table 4-1 are the results obtained with the purely empirical first order algorithm, as well as the semi-empirical algorithm, for which it was assumed that only a subset of the isotopic fission chambers were available. The results using the purely empirical second order algorithm exhibit promise, but are still preliminary at this point in time, and are therefore not shown in Table 4-1. For these particular cases, the isotopic responses corresponding to the unavailable isotopic fission chamber(s) were obtained by scaling and averaging the responses of the other isotopic fission chamber(s). This is described by (15) for the case where it was assumed that neither a ${ }^{239} \mathrm{Pu}$ nor a ${ }^{241} \mathrm{Pu}$ fission chamber was available and (16) for the case for which only a ${ }^{241} \mathrm{Pu}$ fission chamber was unavailable for the used fuel assay. 
Table 4-1: Average RMSE values for total fissile plutonium and ${ }^{235} \mathrm{U}$ mass for simulated assays

\begin{tabular}{|c|c|c|c|c|c|}
\hline Algorithm & $\begin{array}{c}\text { Isotopic } \\
\text { Response } \\
\text { Fission } \\
\text { Chambers }\end{array}$ & $\begin{array}{c}{ }^{235} \text { U RMSE } \\
\text { (NGSI 64) }\end{array}$ & $\begin{array}{c}{ }^{239} \mathrm{Pu}+{ }^{241} \mathrm{Pu} \\
\text { RMSE } \\
\text { (NGSI 64) }\end{array}$ & $\begin{array}{c}{ }^{235} \text { U RMSE } \\
\text { (27 Diversions) }\end{array}$ & $\begin{array}{c}{ }^{239} \mathrm{Pu}+{ }^{241} \mathrm{Pu} \\
\mathrm{RMSE} \\
\text { (27 Diversions) }\end{array}$ \\
\hline Semi-Empirical & $\begin{array}{c}{ }^{235} \mathrm{U}_{,}{ }^{239} \mathrm{Pu}, \\
{ }^{241} \mathrm{Pu}\end{array}$ & $11.2 \%$ & $1.80 \%$ & $4.76 \%$ & $1.44 \%$ \\
\hline Semi-Empirical & ${ }^{235} \mathrm{U},{ }^{239} \mathrm{Pu}$ & $19.24 \%$ & $2.56 \%$ & Not Calculated & Not Calculated \\
\hline Semi-Empirical & ${ }^{235} \mathrm{U}$ & $27.3 \%$ & $4.37 \%$ & Not Calculated & Not Calculated \\
\hline $\begin{array}{c}\text { First Order } \\
\text { Empirical }\end{array}$ & None & $10.5 \%$ & $8.1 \%$ & $3.2 \%$ & $9.5 \%$ \\
\hline
\end{tabular}

$$
\begin{gathered}
x_{i}^{239}(t)=\left[\frac{1}{64} \sum_{j=1}^{64} \frac{x_{j}^{239}(t)}{x_{j}^{235}(t)}\right] \cdot x_{i}^{235}(t) \quad x_{i}^{241}(t)=\left[\frac{1}{64} \sum_{j=1}^{64} \frac{x_{j}^{241}(t)}{x_{j}^{235}(t)}\right] \cdot x_{i}^{235}(t) \\
x_{i}^{241}(t)=\left[\frac{1}{64} \sum_{j=1}^{64} \frac{x_{j}^{241}(t)}{x_{j}^{239}(t)}\right] \cdot x_{i}^{239}(t)
\end{gathered}
$$

\subsection{Application of Algorithms to RPI's Experimental Data}

Experiments were conducted by RPI at their Gaertner Linear Accelerator facility August 1-5, 2011, using the $1.8 \mathrm{~m}$ - edge cubic LSDS shown in Figure 4-4. The pulsed neutrons are generated by the electron LINAC, with $\sim 50 \mathrm{MeV}$ electrons impinging upon a tantalum target, resulting in $(\mathrm{e}, \gamma)$ bremsstrahlung reactions, in turn resulting in $(\gamma, n)$ reactions [19]. These $(\gamma, n)$ reactions provide a neutron energy spectrum peaking around $0.5 \mathrm{MeV}$. Assay targets used in these experiments consisted of combinations of the following items:

1. A fresh $\mathrm{UO}_{2}$ Special Power Excursion Reaction Test (SPERT) fuel pin, having a ${ }^{235} \mathrm{U}$ enrichment of 4.8 atomic percent (35.2 $\mathrm{g}$ of $\left.{ }^{235} \mathrm{U}\right)$.

2. Ten highly enriched $(93.3 \%){ }^{235} \mathrm{U}$ (HEU) discs, with each disc having a diameter of approximately $1.27 \mathrm{~cm}$ and containing between 0.2 and $0.3 \mathrm{~g}$ of ${ }^{235} \mathrm{U}$.

3. A PuBe source containing approximately $96 \mathrm{~g}$ of ${ }^{239} \mathrm{Pu}$

A picture showing the SPERT fuel pin with HEU discs wrapped around the pin in aluminum foil is given in Figure 4-5.

PNNL analyzed the data from these experiments, using the first and second order empirical models, as well as the semi-empirical model. The calibration set for the first and second order empirical models is shown in Table 4-2. The PuBe source was the only item that contained ${ }^{239} \mathrm{Pu}$, so that there were effectively only 2 distinct ${ }^{239} \mathrm{Pu}$ mass quantities, 0 and $96 \mathrm{~g}$. As a result, only the ${ }^{235} \mathrm{U}$ mass fitting used a quadratic term when applying the second order empirical model to determine the masses of ${ }^{239} \mathrm{Pu}$ and 
${ }^{235} \mathrm{U}$. The relative errors in the estimates of the ${ }^{235} \mathrm{U}$ masses are given in Table 4-3. The "Fuel Pin +3 HEU discs" and "Fuel Pin + 10 HEU discs" cases were each had duplicate data sets from repeated experiments. Therefore, one set of data was used in the calibration, as indicated in Table 4-2, and the other set was used in the test set, shown in Table 4-3. For the semi-empirical algorithm, the signal from the ${ }^{238} \mathrm{U}$ fission chamber that was inserted into the LSDS assay chamber along with the assay targets was used as the $Y(t)$ in (9), and the ${ }^{235} \mathrm{U}$ fission chamber signal was used in place of $X(t)$ in (8). Since RPI's ${ }^{239} \mathrm{Pu}$ fission chamber was not used for this set of experiments, only the ${ }^{235} \mathrm{U}$ masses were estimated using the semi-empirical algorithm. The calibration set used for the semi-empirical algorithm is given in Table 4-4, and the relative errors for the ${ }^{235} \mathrm{U}$ mass estimates for the test set cases are given in Table 4-5. As shown in Table 4-5, the semi-empirical algorithm underestimated the true ${ }^{235} \mathrm{U}$ mass in all cases. The RMSE in the ${ }^{235} \mathrm{U}$ mass over 5 of the 11 cases was $4.2 \%$ using the semi-empirical algorithm, for which the remaining 6 cases were used as the calibration set. The overall RMSE values in the ${ }^{235} \mathrm{U}$ mass for the first and second order empirical model were $4.6 \%$ and $2.3 \%$, respectively. Since only two distinct masses of ${ }^{239} \mathrm{Pu}$ were used in the experiments, it is difficult to evaluate the performance of the empirical algorithms on estimating ${ }^{239} \mathrm{Pu}$ mass.

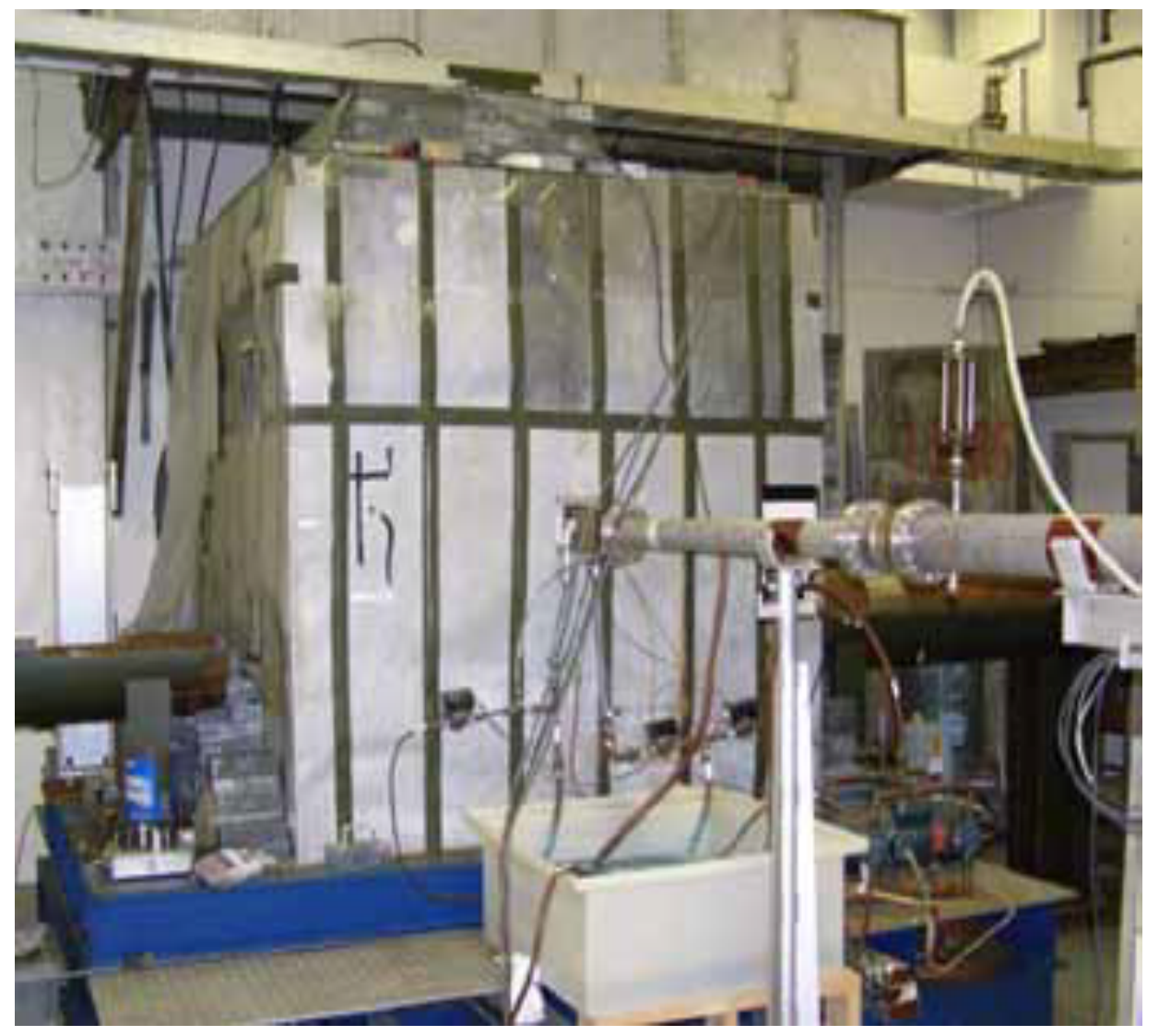

Figure 4-4: LSDS at the RPI Gaertner Linear Accelerator 


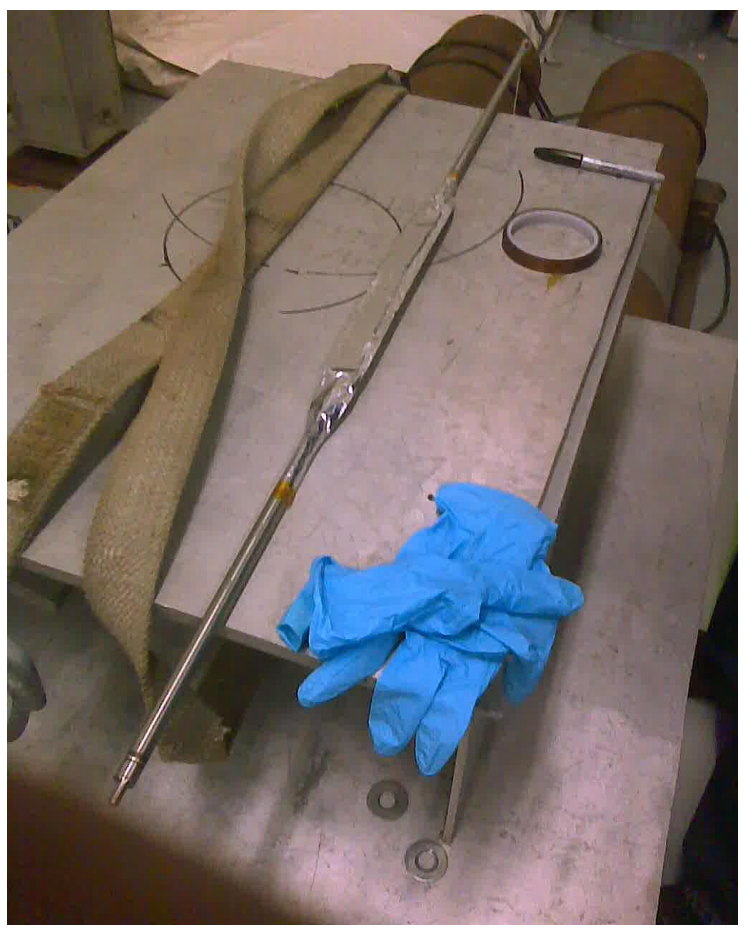

Figure 4-5: HEU discs wrapped in aluminum foil around the fresh SPERT fuel pin

Table 4-2: Calibration set for first and second order empirical algorithms for experimental data

\begin{tabular}{|c|c|c|}
\hline Assay Target & ${ }^{235} \mathrm{U}$ mass & ${ }^{239}$ Pu mass \\
\hline Fuel Pin & $35.20 \mathrm{~g}$ & $0 \mathrm{~g}$ \\
\hline Fuel Pin + 3 HEU discs & $35.99 \mathrm{~g}$ & $0 \mathrm{~g}$ \\
\hline Fuel Pin + 10 HEU discs & $37.83 \mathrm{~g}$ & $0 \mathrm{~g}$ \\
\hline Fuel Pin + PuBe Source + 3 HEU discs & $35.99 \mathrm{~g}$ & $96 \mathrm{~g}$ \\
\hline PuBe Source & $0 \mathrm{~g}$ & $96 \mathrm{~g}$ \\
\hline 3 HEU discs & $0.79 \mathrm{~g}$ & $0 \mathrm{~g}$ \\
\hline
\end{tabular}

Table 4-3: Relative errors for ${ }^{235} \mathrm{U}$ mass estimates for the first and second order empirical algorithms

\begin{tabular}{|c|c|c|c|}
\hline Assay Target & $\begin{array}{c}\text { True }{ }^{235} \mathrm{U} \\
\text { Mass }\end{array}$ & $\begin{array}{c}1^{\text {st }} \text { order Empirical Algorithm: } \\
\text { Relative Error }\end{array}$ & $\begin{array}{l}2^{\text {nd }} \text { order Empirical Algorithm: } \\
\text { Relative Error }\end{array}$ \\
\hline Fuel Pin & $35.20 \mathrm{~g}$ & $-8.3 \%$ & $-3.2 \%$ \\
\hline Fuel Pin + 1 HEU disc & $35.46 \mathrm{~g}$ & $-2.7 \%$ & $-2.6 \%$ \\
\hline Fuel Pin + 3 HEU discs & $35.99 \mathrm{~g}$ & $1.2 \%$ & $1.9 \%$ \\
\hline Fuel Pin + 5 HEU discs & $36.49 \mathrm{~g}$ & $3.1 \%$ & $0.0 \%$ \\
\hline Fuel Pin + 10 HEU discs & $37.83 \mathrm{~g}$ & $5.7 \%$ & $3.9 \%$ \\
\hline Fuel Pin + PuBe Source & $35.20 \mathrm{~g}$ & $5.0 \%$ & $-0.6 \%$ \\
\hline $\begin{array}{c}\text { Fuel Pin }+ \text { PuBe Source }+1 \\
\text { HEU disc }\end{array}$ & $35.46 \mathrm{~g}$ & $-1.2 \%$ & $0.2 \%$ \\
\hline
\end{tabular}


Table 4-4: Calibration set for the semi-empirical algorithm for experimental data.

\begin{tabular}{|c|c|c|}
\hline Assay Target & ${ }^{235} \mathrm{U}$ mass & ${ }^{239}$ Pu mass \\
\hline Fuel Pin & $35.20 \mathrm{~g}$ & $0 \mathrm{~g}$ \\
\hline Fuel Pin + 3 HEU discs & $35.99 \mathrm{~g}$ & $0 \mathrm{~g}$ \\
\hline Fuel Pin + 10 HEU discs & $37.83 \mathrm{~g}$ & $0 \mathrm{~g}$ \\
\hline Fuel Pin + PuBe Source & $35.20 \mathrm{~g}$ & $96 \mathrm{~g}$ \\
\hline Fuel Pin + PuBe Source + 1 HEU disc & $35.46 \mathrm{~g}$ & $96 \mathrm{~g}$ \\
\hline Fuel Pin + PuBe Source + 3 HEU disc & $35.99 \mathrm{~g}$ & $0 \mathrm{~g}$ \\
\hline
\end{tabular}

Table 4-5: Relative errors for ${ }^{235} \mathrm{U}$ mass estimates using the semi-empirical algorithm.

\begin{tabular}{|c|c|c|}
\hline Assay Target & True $^{\mathbf{2 3 5}} \mathbf{U}$ Mass & Relative Error \\
\hline Fuel Pin & $35.20 \mathrm{~g}$ & $-7.1 \%$ \\
\hline Fuel Pin + 1 HEU disc & $35.46 \mathrm{~g}$ & $-5.7 \%$ \\
\hline Fuel Pin + 3 HEU discs & $35.99 \mathrm{~g}$ & $-1.6 \%$ \\
\hline Fuel Pin + 5 HEU discs & $36.49 \mathrm{~g}$ & $-1.1 \%$ \\
\hline Fuel Pin + 10 HEU discs & $37.83 \mathrm{~g}$ & $-0.2 \%$ \\
\hline
\end{tabular}

The statistics of the experiment measurements may present challenges to the algorithm. The poorer statistics introduce sharp artificial features (i.e., spikes) in the fission chamber signals that are more difficult to fit using basis vectors representing a wide range of parameters. The measured ${ }^{238} \mathrm{U}$ assay signal is shown in Figure 4-6 along with the fit approximated with the 1st order algorithm for the case of the SPERT fuel pin + PuBe source and 1 HEU disc. Inspecting this graph, it is likely that if the statistics were better, such as by using more fission chamber detectors or a longer assay time, the relative errors in the mass estimates would be smaller. 


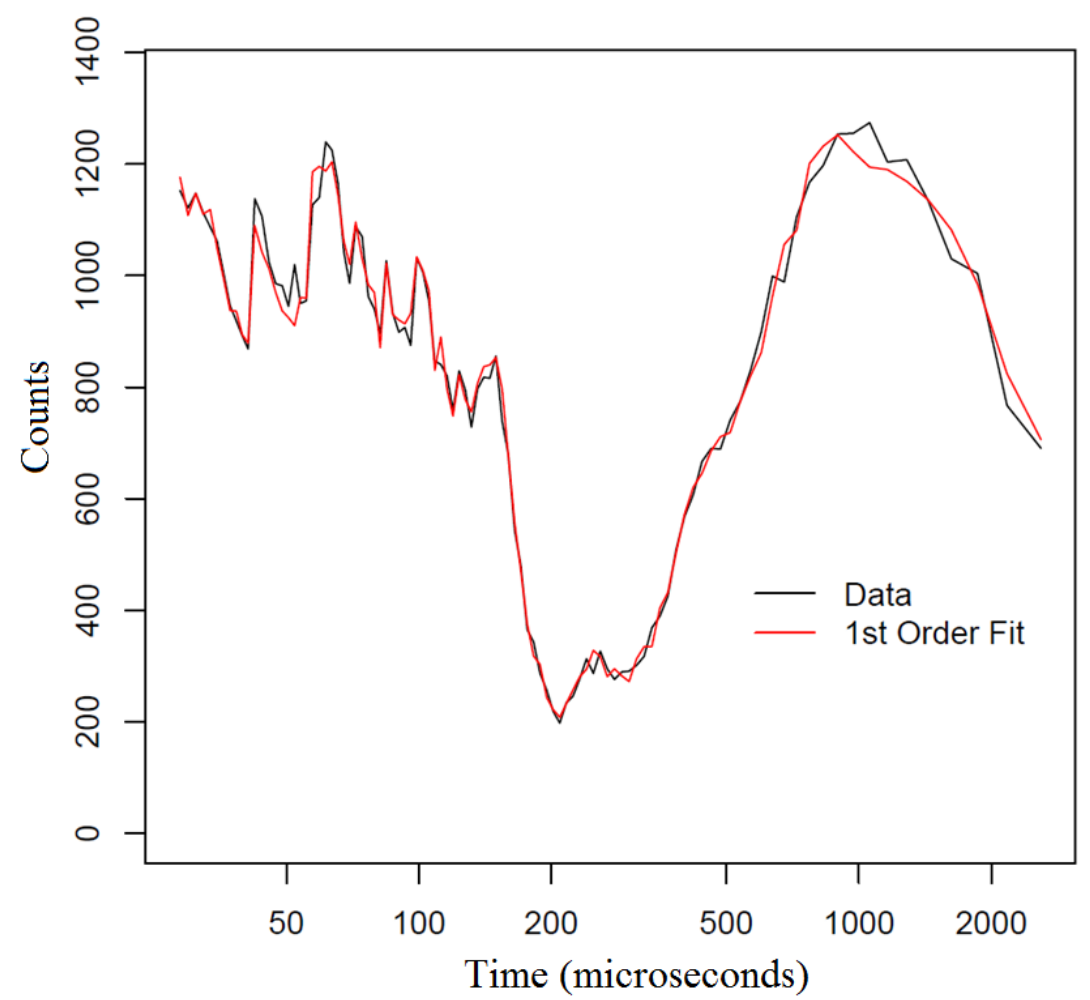

Figure 4-6: Measured ${ }^{238} \mathrm{U}$ assay signal and corresponding fit for the assay of a SPERT fuel pin, PuBe source, and $1 \mathrm{HEU}$ disc 


\subsection{LSDS and Semi-Empirical Algorithm Sensitivity Analyses}

In addition to quantifying the performance of the semi-empirical algorithm by measuring how accurately this algorithm could be used to determine fissile mass in both the simulations and experiments, a number of sensitivity studies were performed on the semi-empirical algorithm. These studies include sensitivity of the errors in mass estimates to the size of calibration set, uncertainty in calibration set masses, and range of neutron slowing-down times used in the algorithm. Sensitivity studies were also conducted on the LSDS instrument as a whole: the ability to detect fissile mass within the central depths of the usedfuel assemblies, the ability to detect changes in fissile mass within a single fuel pin, and the effect of various amounts of water added to the fuel pins.

\subsection{Sensitivity of the Semi-Empirical Algorithm to Uncertainties in Calibration Set Masses}

All of the models studied this year rely on a calibration set to train the algorithm. In practice, this would occur by conducting LSDS measurements on a used fuel assembly, and then conducting destructive analysis to determine the masses in the assembly. The masses determined from these analyses will be determined with some non-zero uncertainty. An analysis was performed to determine the sensitivity of the RMSE to uncertainties in the calibration set masses. For this analysis, the following procedure was repeated 1000 times for standard deviations of $0 \%, 1 \%, 2 \%$, and $5 \%$ in the calibration masses. First, the calibration set masses were sampled from a lognormal distribution having a particular standard deviation $(\sigma)$. Then, the masses of the NGSI 64 fuel assembly library were calculated as described in section 3.1 using these "noisy" calibration set masses. For each set of 1000 trials with a given standard deviation, the RMSE between the calculated and true masses over the 64 NGSI assemblies was calculated. The average over these 1000 RSME values is shown in Figure 5-1 for the NGSI 64. This calculation was repeated for the 27 diversion assemblies, for which the results are shown in Figure 5-2. For a 2\% standard deviation level of uncertainty in the calibration set masses, the RMSE for the sum of the ${ }^{239} \mathrm{Pu}$ and ${ }^{241} \mathrm{Pu}$ masses is still less than $3 \%$ for both of these assembly sets. Therefore, the accuracy of the calculated masses is still acceptable allowing for realistic uncertainties of the calibration set masses. 


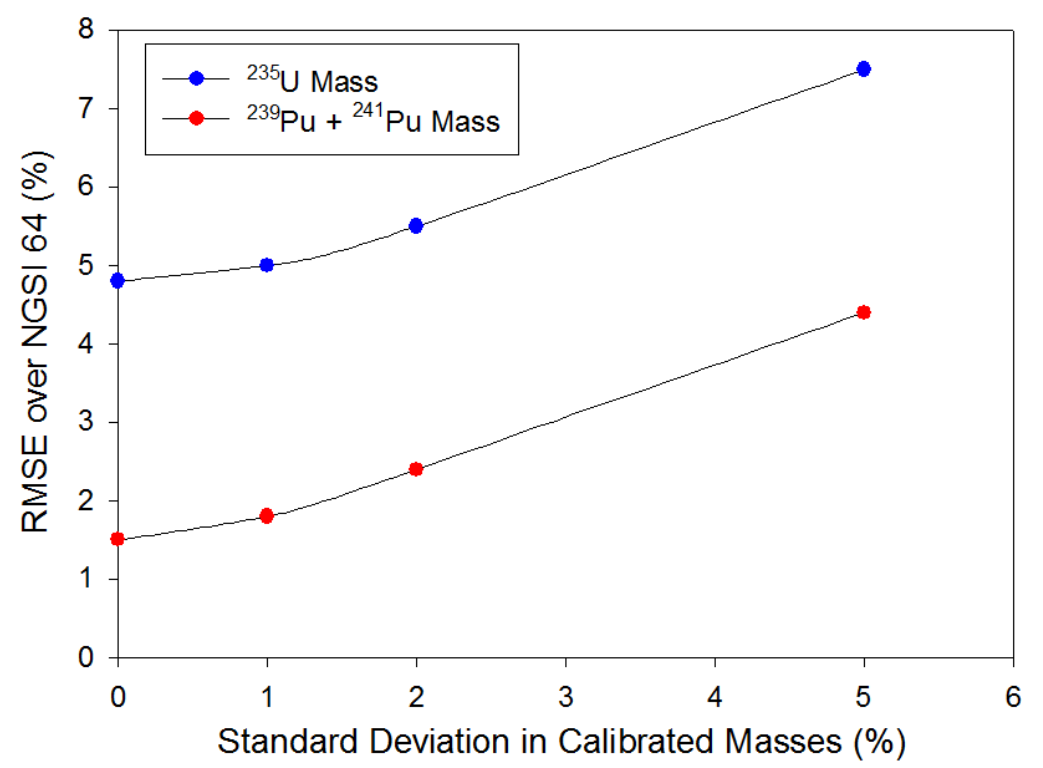

Figure 5-1: Results for ${ }^{235} \mathrm{U}$ and ${ }^{239} \mathrm{Pu}+{ }^{241} \mathrm{Pu}$ mass estimates applying the semi-empirical algorithm on MCNPX-simulated assay of the NGSI 64

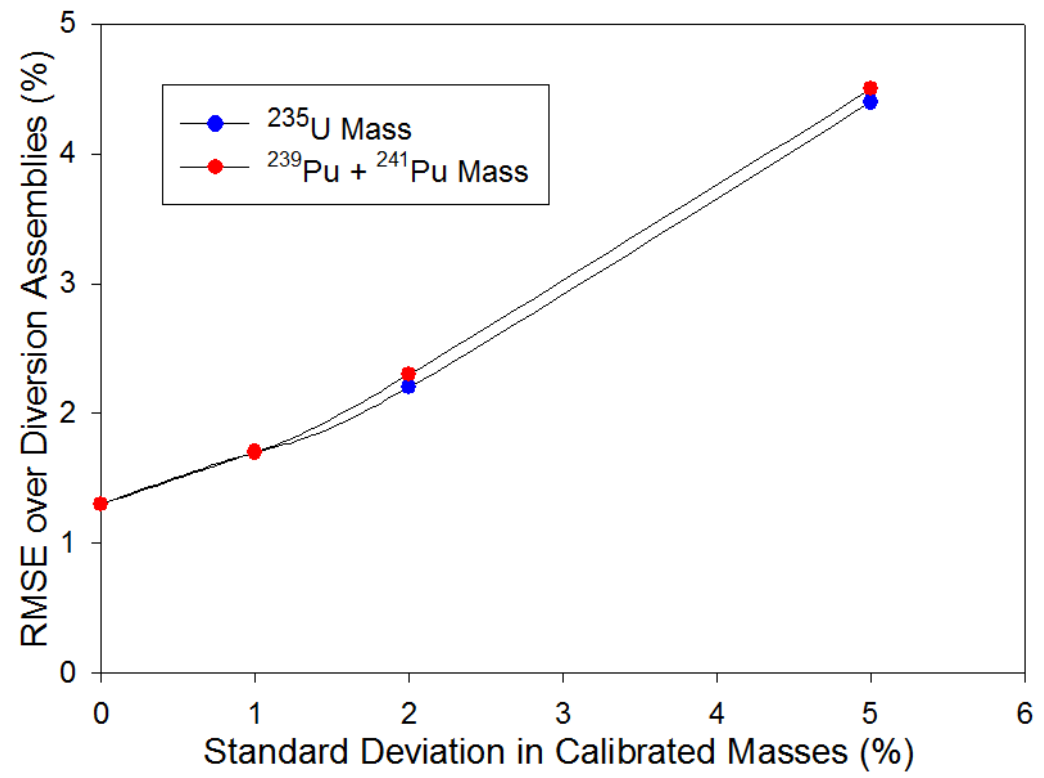

Figure 5-2: Results for ${ }^{235} \mathrm{U}$ and ${ }^{239} \mathrm{Pu}+{ }^{241} \mathrm{Pu}$ mass estimates applying the semi-empirical algorithm on MCNPX-simulated assay of the 27 diversion assembly models

\subsection{Sensitivity to Number of Fuel Assemblies in Calibration Set}

An analysis was performed to determine the effect of the number of calibration fuels used in the semiempirical algorithm on the errors in the fissile isotopic mass estimates over the NGSI 64. The results of this analysis are shown in Figure 5-3. The effect of the calibration set size is not very pronounced for the ${ }^{235} \mathrm{U}$ mass estimates, however, the ${ }^{239} \mathrm{Pu}$ mass estimates improve monotonically with increasing 
calibration set size. Assuming the entire NGSI 64 library as the calibration set to generate the basis vectors to span the self-shielding functions of these same 64 fuels is akin to assuming an infinite calibration set size. Such a case may be realized if the MCNPX simulation models and cross section libraries used by MCNPX were perfect. At present, this is not realistic, and so in the foreseeable future, experimental calibrations using well-characterized used-fuel assemblies will be required to achieve reasonable uncertainties in fissile isotopic mass estimates using these empirical algorithms.

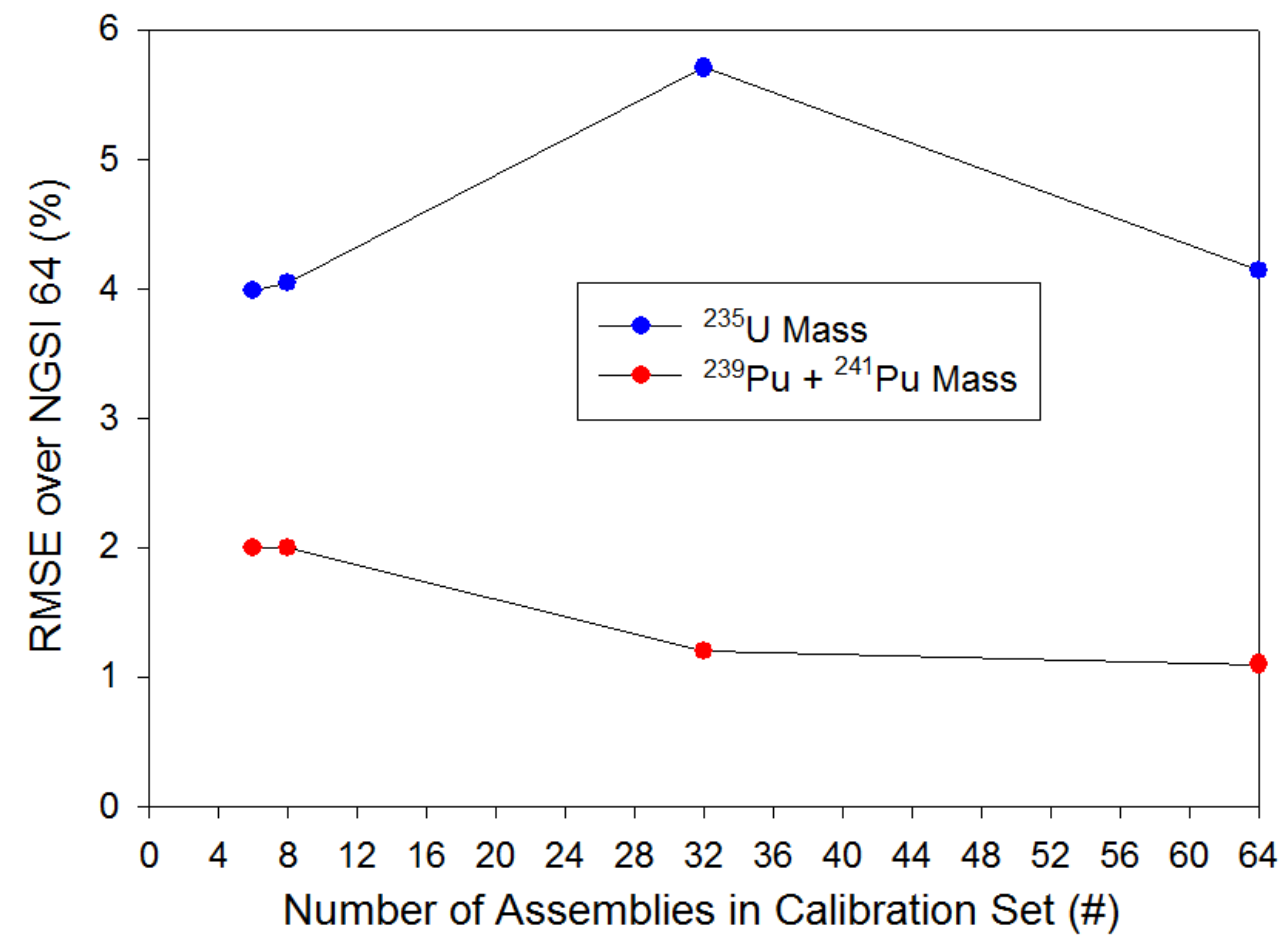

Figure 5-3: Results for ${ }^{235} \mathrm{U}$ and ${ }^{239} \mathrm{Pu}+{ }^{241} \mathrm{Pu}$ mass estimates for various calibration set sizes

\subsection{Sensitivity to Range of Neutron Slowing-Down Times Used in Analysis}

Currently, the neutron slowing-down time region examined in the algorithms is between 20 and $2000 \mu$ s. This range is used in all of the algorithms. One measurement concern is that the fission chamber detectors will not be able to recover from the initial gamma-ray flash until after a few tens of microseconds. An analysis was performed in which time regions of $60 \mu \mathrm{s}$ to $2000 \mu \mathrm{s}, 336 \mu \mathrm{s}$ to $2000 \mu \mathrm{s}$, and $1125 \mu$ s to $2000 \mu \mathrm{s}$ were used in the semi-empirical algorithm. These time regions were chosen since they correspond to the coarse time bins over which the Monte Carlo variance reduction weight-windows were applied in the simulations. The RMSE values of the isotopic masses using these time regions in the semi-empirical algorithm are shown in

Table 5-1, averaged over the 58 NGSI fuels that are not part of the calibration set. The RMSE values become increasingly poorer for both the ${ }^{235} \mathrm{U}$ and total fissile plutonium mass as more of the earlier times are neglected in the analysis. The data in the time window from 60 to 336 is clearly critical to an accurate 
extraction of the plutonium masses. The reason for the sharp drop in RMSE going from the $20 \mu \mathrm{s}-$ $2000 \mu$ s to the $60 \mu \mathrm{s}-2000 \mu$ s time range is unknown.

Table 5-1: Average RMSE values obtained for ${ }^{235} \mathrm{U}$ and ${ }^{239} \mathrm{Pu}+{ }^{241} \mathrm{Pu}$ masses in the NGSI 64 using various slowing-down time ranges in the semi-empirical algorithm

\begin{tabular}{|c|c|c|}
\hline Time Range $(\boldsymbol{\mu s})$ & RMSE of ${ }^{\mathbf{2 3 5}} \boldsymbol{U}$ Mass & RMSE of ${ }^{\mathbf{2 3 9}} \mathbf{P u}+{ }^{\mathbf{2 4 1}} \mathrm{Pu}$ Mass \\
\hline $20-2000$ & $11.2 \%$ & $1.8 \%$ \\
\hline $60-2000$ & $4.4 \%$ & $2.1 \%$ \\
\hline $336-2000$ & $39.9 \%$ & $11.7 \%$ \\
\hline $1125-2000$ & $241 \%$ & $34.9 \%$ \\
\hline
\end{tabular}

\subsection{LSDS for Full Volume Assay Capability}

One of the advantages of the LSDS technique over other assay techniques is the potential to be sensitive to the entire fuel assembly, not just the outer components. It was anticipated that due to self-absorption, there would be less sensitivity to inner portions of the assembly. Calculations were conducted to quantify this reduced sensitivity toward the middle of the assembly. To investigate this issue, the tally tagging feature of MCNPX 2.7.0 [17] was used. This feature enables more detailed output for the simulation results.

For this calculation, an assay of the assembly having $30 \mathrm{GWd} / \mathrm{MTU}$ burnup, $3 \mathrm{wt} \%$ initial enrichment of ${ }^{235} \mathrm{U}$, and 20 years of cooling time was simulated. The assay signal observed in the ${ }^{238} \mathrm{U}$ fission chamber was tallied as a function of row, from the central to outer concentric rows of the assembly, where each row is defined as a square of fuel pins, as shown in the inset in Figure 5-4. The interrogation neutron flux was also tallied as a function of row. The results, normalized to 1.0 for the outer row, are shown in Figure 5-4. For this fuel assembly, the interrogation neutron flux in the center row is $\sim 90 \%$ that of the outer row. The assay signal per assembly row is a direct measure of the sensitivity of the LSDS to the fissile mass in the assembly as a function of depth. The LSDS is able to measure the fissile mass in the inner row of the assembly $\sim \%$ as effectively as the outer row. While there is less sensitivity to the middle of the assembly, there remains significant sensitivity. It should be noted that the $55 \%$ reduced sensitivity is likely an underestimate due to how the assay signal was scored. For instances, fission neutrons that originate in the center of the assembly, but then generate additional fission neutrons in the outer edges of the assembly, will be tallied as if the "observed" fission neutrons originated in the outer edge of the assembly.

The results shown in Figure 5-4 are encouraging, since they suggest that the performance of the LSDS may be improved by placing additional detectors in the instrumentation tubes in the PWR assembly. A similar investigation conducted by RPI indicated significantly more self-shielding [18], although the RPI investigation was performed on fresh fuel having a much higher enrichment of $9.8 \mathrm{wt} \%{ }^{235} \mathrm{U}$. 


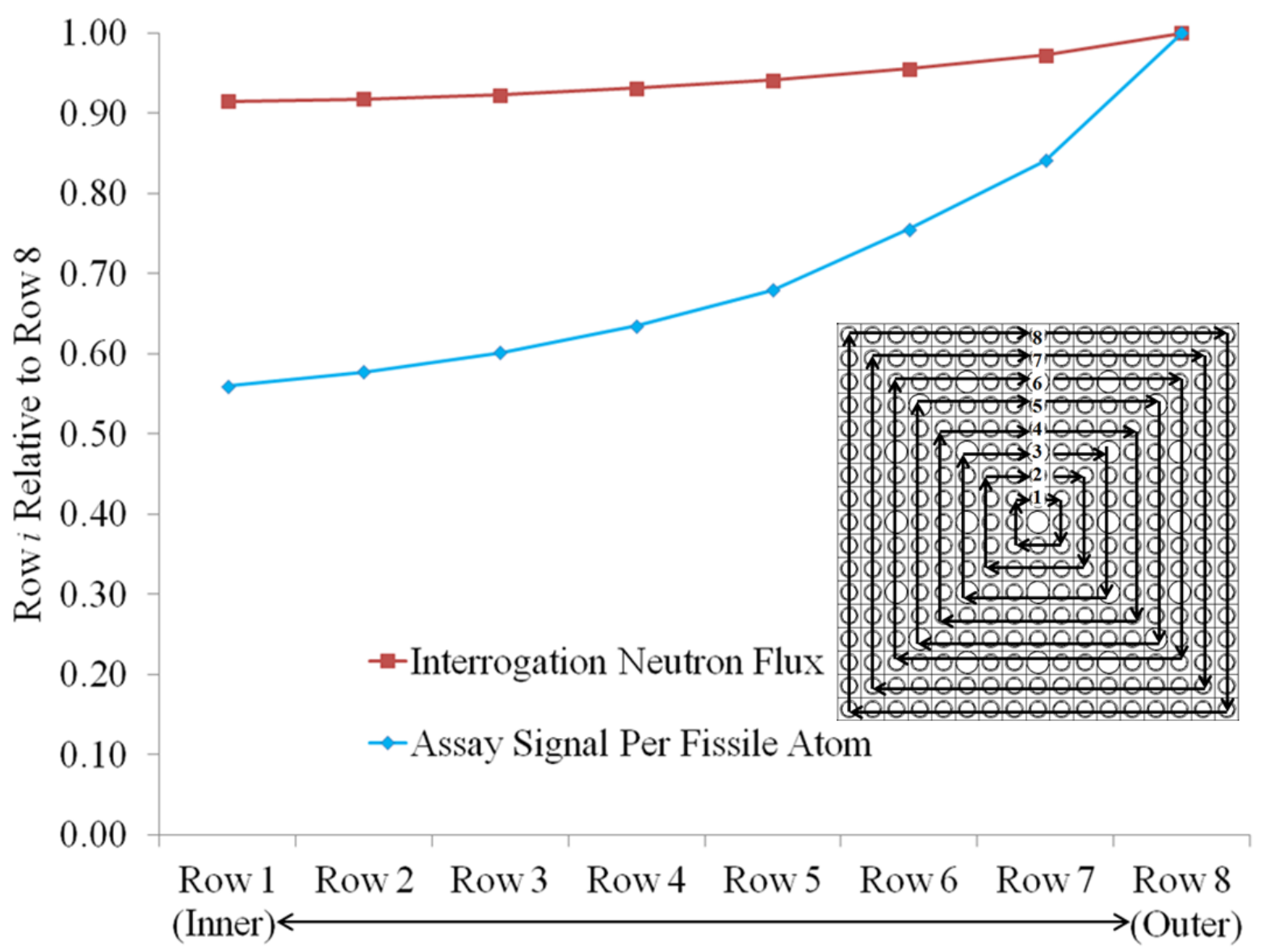

Figure 5-4: Assay signal per total number of ${ }^{235} \mathrm{U},{ }^{239} \mathrm{Pu}$, and ${ }^{241} \mathrm{Pu}$ atoms as a function of depth from the inner to the outer rows of fuel pins of the assembly. Also shown is the interrogation neutron flux as a function of depth in the assembly.

\subsection{Assays of Single and Nine Fresh Fuel Pin Arrays}

The assays of single fresh fuel pins and nine fuel pin arrays were also simulated using the LSDS model in MCNPX. The assay signal results for ${ }^{238} \mathrm{U}$ fission chamber signal for the simulated assay of single fresh fuel pins having various $w t \%$ enrichment of ${ }^{235} \mathrm{U}$ are shown in the left-hand graph of Figure 5-5. In the right-hand graph, the ratio of the assay signals from the $2 \mathrm{wt} \%$ to $5 \mathrm{wt} \%$ enriched fuel pin relative to the $1 \mathrm{wt} \%$ enriched fuel pin are shown. The assay signals are not proportional to the ${ }^{235} \mathrm{U}$ (fissile material) present in the fresh fuel pins, indicating the effect of self-shielding even for a single pin. Similar results are shown in Figure 5-6 for the simulated assays of nine pin arrays of fresh fuel having various ${ }^{235} \mathrm{U}$ enrichments. From the FY2011 analysis report [16], it was mentioned that such results would be analyzed to determine if improvements could be made to the analytical self-shielding model. These attempts were unsuccessful, since the self-shielding effects could not be easily characterized within the context of the analytical model and extended to full assemblies. 

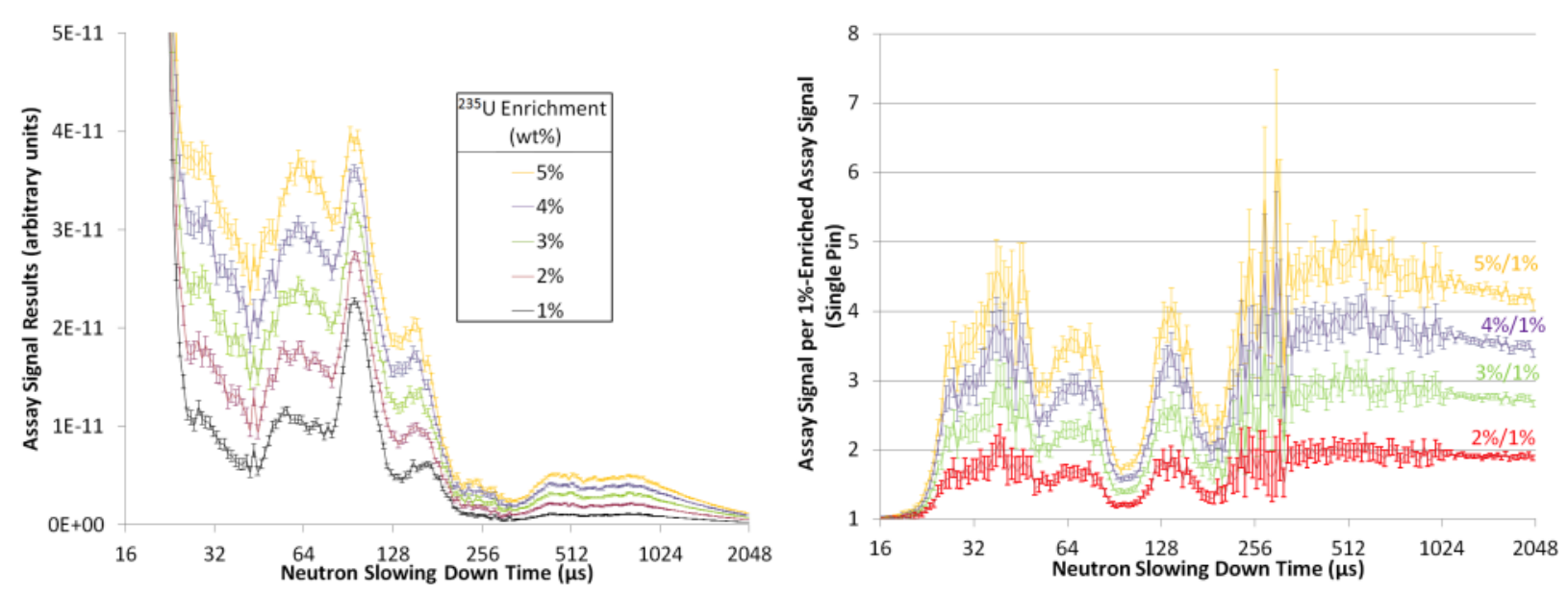

Figure 5-5: LEFT: ${ }^{238} \mathrm{U}$ fission chamber signal obtained from the simulated assay of single fresh fuel pins of various $\mathrm{wt} \%$ of ${ }^{235} \mathrm{U}$ enrichments. RIGHT: The ${ }^{238} \mathrm{U}$ fission chamber signal are shown normalized to the signal for the $1 \mathrm{wt} \%$ enriched fuel pin.
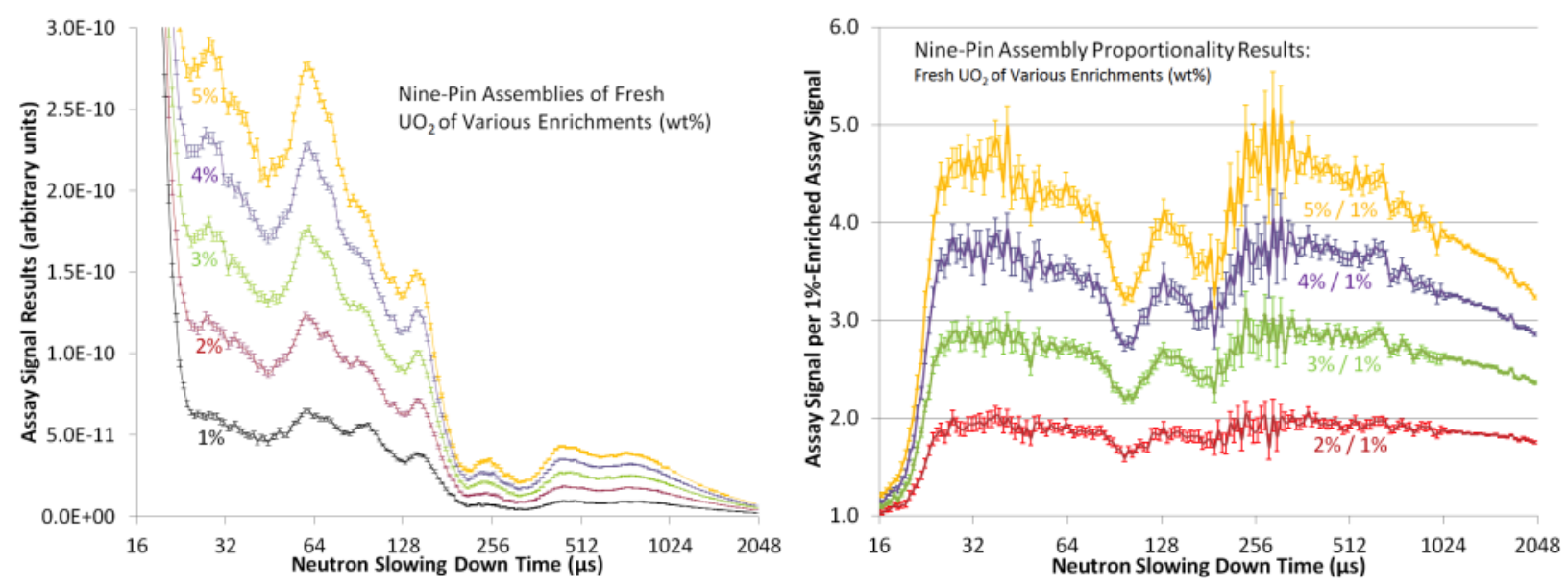

Figure 5-6: LEFT: ${ }^{238} \mathrm{U}$ fission chamber signal obtained from the simulated assay of nine fresh fuel pin arrays of various $\mathrm{wt} \%$ of ${ }^{235} \mathrm{U}$ enrichments. RIGHT: The ${ }^{238} \mathrm{U}$ fission chamber signal signals are shown normalized to the signal for the $1 \mathrm{wt} \%$ enriched nine fuel pin array.

\subsection{Sensitivity to Water in Fuel Pins}

A sensitivity analysis was also performed using MCNPX simulations to determine the effect on the ${ }^{238} \mathrm{U}$ fission chamber signal when adding various amounts of water to the central row of fuel pins. For this study, the NGSI fuel assembly having $30 \mathrm{GWd} / \mathrm{MTU}, 3 \mathrm{wt} \%$ initial ${ }^{235} \mathrm{U}$ enrichment, and 20 years of cooling time was used as the base assembly model. The goal of this analysis was to determine whether cracked fuel pins could be detected using the LSDS. The results of this analysis are shown in Figure 5-7. While the water clearly has an effect on the assay signal, no attempt has been made to quantify the impact of the water on the algorithms as calibrated against the NGSI-64 set of assemblies. 


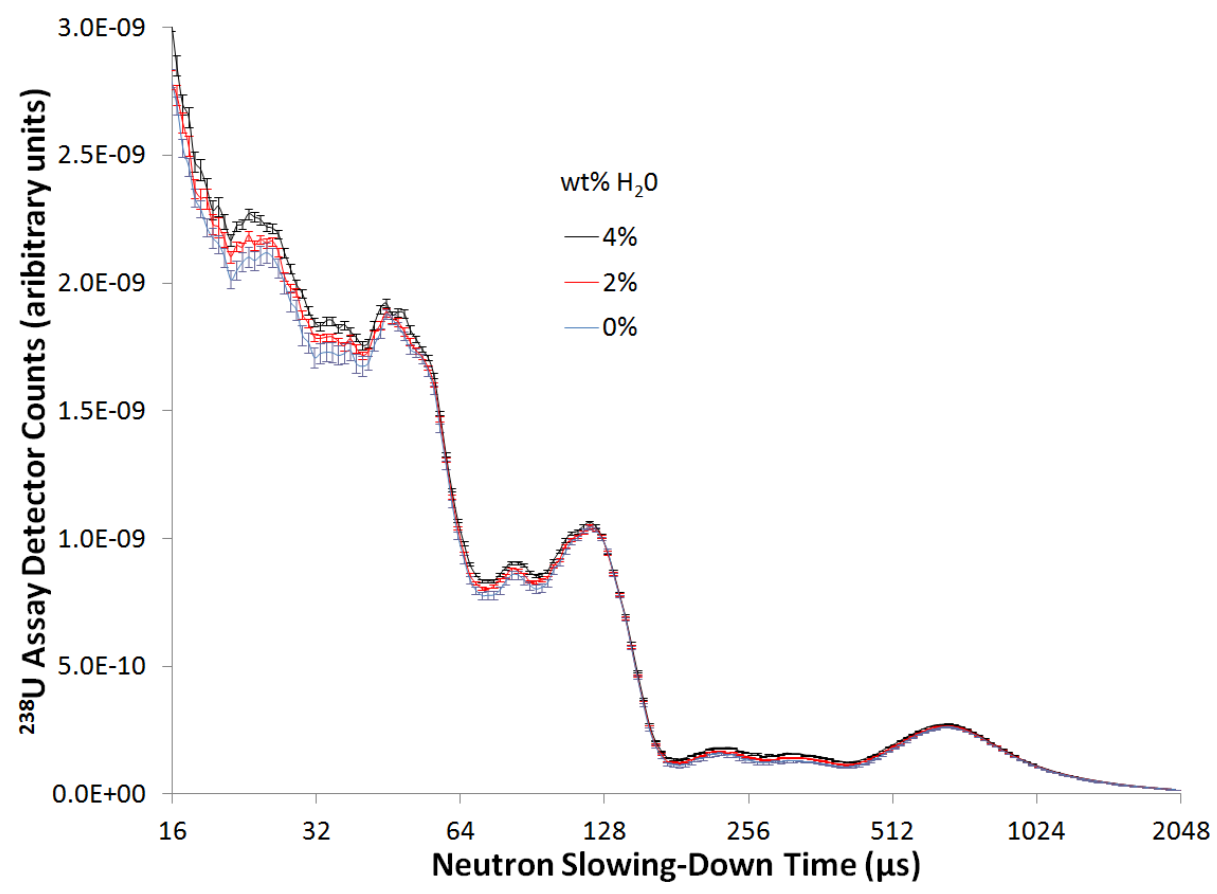

Figure 5-7: Simulated ${ }^{238} U$ fission chamber signals for various $w t \%$ of water of each pin in the central row of fuel pins in the $30 \mathrm{GWd} / \mathrm{MTU}, 3 \mathrm{wt} \%$ enriched, 20 year cooling time assembly 


\subsection{Conceptual LSDS Design Studies}

Ongoing efforts include developing a conceptual design of an LSDS for use in assaying used-fuel assemblies. Assay times of approximately half of an hour are considered reasonable for the LSDS. In FY2012, simulation analyses were conducted to determine the required neutron source strength to achieve this assay time. Also, an analysis was conducted to determine the size and dimensions of the lead required for the LSDS.

\subsection{Neutron Source Strength Requirements}

The number of source neutrons required in a notional LSDS to achieve the same level of uncertainties in the results of the current MCNPX simulations are shown in Figure 6-1. These results were calculated assuming the absolute detection efficiency of $32{ }^{238} \mathrm{U}$ fission chambers combined. Each of these fission chambers were assumed to have cylindrical dimensions of $60 \mathrm{~cm}$ high and $3.29 \mathrm{~cm}$ in diameter coated with an active layer of $1 \mathrm{mg} / \mathrm{cm}^{2}$ of pure ${ }^{238} \mathrm{U}$ metal. A total source neutron value of $10^{16}$ neutrons is currently assumed for generating Poisson noise in the simulated fission chamber signals for the time spectra analysis algorithms used to estimate the fissile isotopic masses. This number of neutrons can be reasonably generated within a 30 minute time period using currently available LINACs. The stair-step behavior of this figure is created by the variance reduction techniques applied in the simulation. The MCNPX simulations provide statistical uncertainties smaller than the $10^{16}$ neutrons only for times greater than $1 \mathrm{~ms}$.

The current statistical uncertainties of the simulations present a challenge for interpretation of some of the results. In the algorithm, it is assumed that the simulated signals are effectively noise free, so that noise equivalent to $10^{16}$ neutrons is added to the signals. When the MCNPX simulations have greater statistical uncertainty than the Poisson noise characteristic of $10^{16}$ neutrons, the simulation uncertainties dominate the analysis, which could create artifacts in the analysis. This situation complicates the interpretation of the analyses. For instance, if the geometry is modified in a manner to effectively increase the number of source neutrons for times less than $1 \mathrm{~ms}$, it is unclear whether the modified geometry provides greater sensitivity, or if the reduced uncertainties are simply a result of improved statistics. A thorough study of the impact of statistical uncertainties on the analysis conclusions would involve varying the statistical precision of the MCNPX simulations within ranges currently supported by computational resources at PNNL and examining the effects of improved precision on convergence of the analysis results. Although this study has not yet been carried out, results to date are encouraging. The analysis is already achieving acceptable uncertainties when Poisson noise characteristic of $10^{16}$ sources neutrons is added to the time spectra, despite significant portions of the time spectra containing statistical noise for far fewer neutrons. Improved statistical noise of the MCNPX simulations may enable reduced uncertainties or reduced number of required source neutrons. 


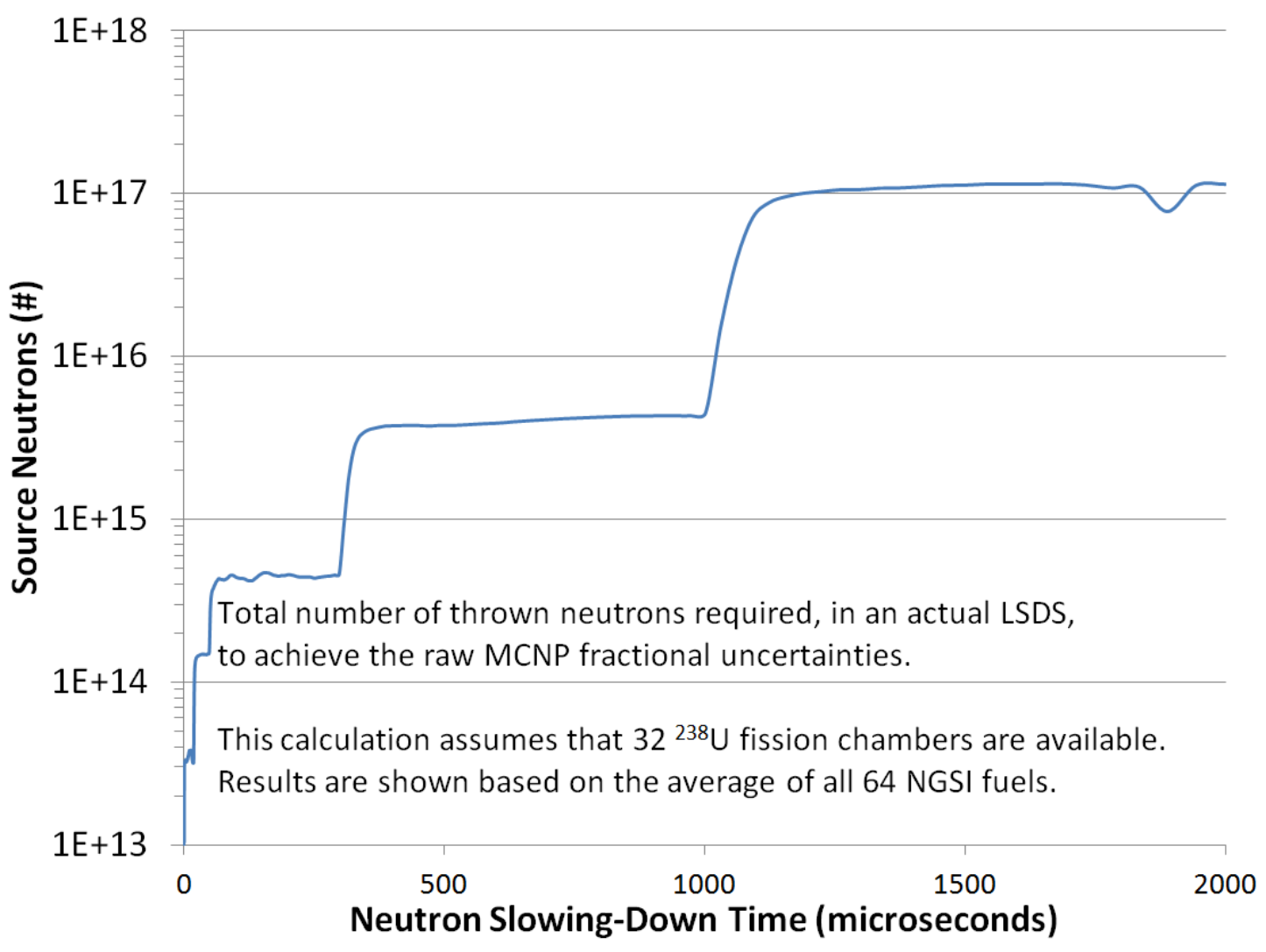

Figure 6-1: Number of source neutrons required in an LSDS to achieve the same level of fractional uncertainties in the MCNPX-simulated ${ }^{238} \mathrm{U}$ fission chamber signal

\subsection{Lead Size Requirements}

An analysis was conducted to determine the appropriate outer dimensions of lead required for the LSDS. For this analysis, cylindrical LSDS instruments having various sizes of lead (outer dimensions) were simulated with the $30 \mathrm{GWd} / \mathrm{MTU}, 3 \mathrm{wt} \%$ initial enrichment, and 20 year cooling time assembly.

For the first round of simulations, the source location was kept constant at approximately $60 \mathrm{~cm}$ from the edge of the fuel assembly. The neutron source was assumed to be isotropic. The results of these simulations are shown in Figure 6-2. These results indicate the cylindrical LSDS having outer dimensions of $1.5 \mathrm{~m}$ height and $1.5 \mathrm{~m}$ diameter, with a mass of 28.6 metric tons, is the smallest LSDS that can achieve a reasonable time-spectral resolution. Increasing the lead outer dimensions to $1.75 \mathrm{~m}$ by $1.75 \mathrm{~m}$ significantly increases the assay signal, as does increasing to the $2 \mathrm{~m}$ by $1 \mathrm{~m}$ size, which are the dimensions of the LSDS that was used for all MCNPX simulations and analyses conducted by PNNL. An interesting feature of the graph in Figure 6-2 is the apparent increase in resolution with increasing LSDS size. One explanation for this would be that fast neutrons which would have otherwise quickly escaped in a smaller LSDS are more likely to scatter, lose energy, and contribute to fissions in the fuel assembly in the larger LSDS. Keeping in mind that very good accuracies on total fissile plutonium were obtained using simulated results of the $2 \mathrm{~m}$ by $1 \mathrm{~m}$ LSDS and the results shown in Figure 6-2, it is likely that $30-40$ metric tons of lead would be sufficient for the LSDS design. At this point, these results on the dimensions of the lead are qualitative. The appropriate way to understand the impact of the lead 
dimensions is to run the process for all geometries for all assemblies of the NGSI 64 set and determine the mass uncertainties.

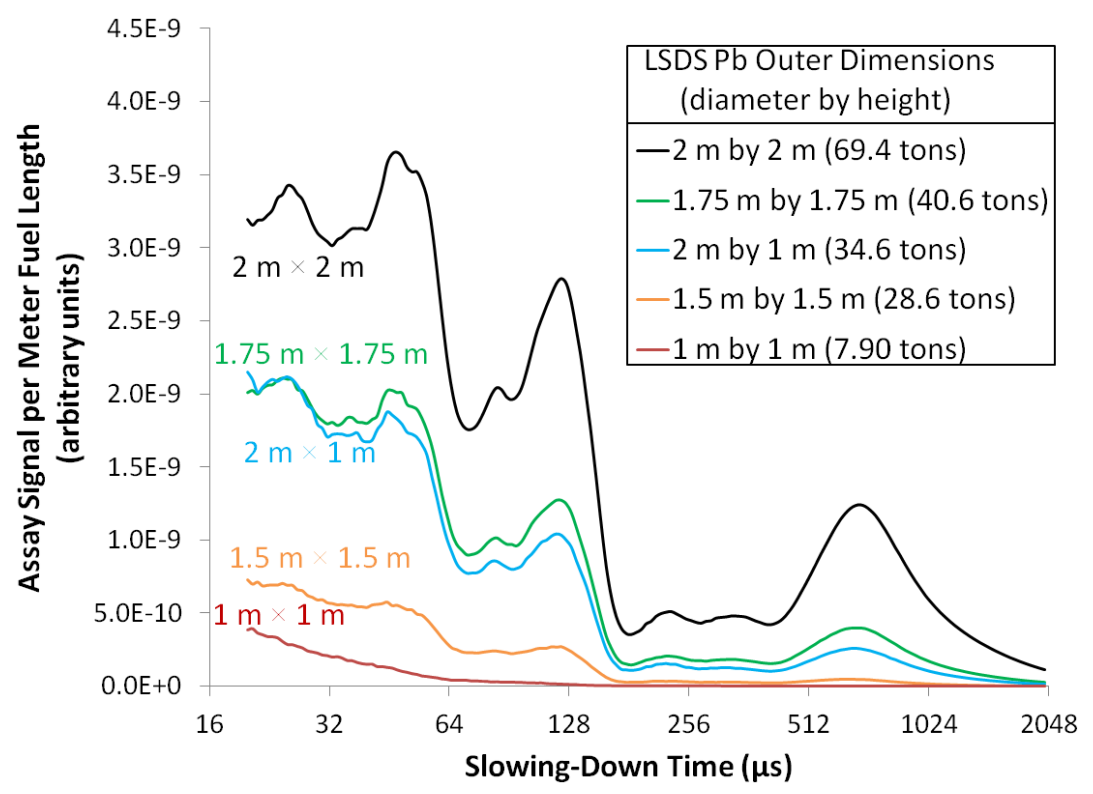

Figure 6-2: Simulated ${ }^{238} \mathrm{U}$ fission chamber signal for cylindrical LSDS instruments of various size (lead outer dimensions). The source location was kept constant at $\sim 60 \mathrm{~cm}$ from the fuel.

Results are shown in Figure 6-3 for the LSDS instruments with different source-to-fuel distances. Results from two lead dimensions, $1.5 \mathrm{~m}$ by $1.5 \mathrm{~m}$ and $1.75 \mathrm{~m}$ by $1.75 \mathrm{~m}$, with the source located at 40 and $60 \mathrm{~cm}$ from the closest edge of the fuel are shown in this figure. The neutron source is assumed to be isotropic in this study. These results show a large increase in signal strength and time-spectral resolution when the source is moved deeper in to the LSDS and closer to the fuel. One cause for this effect may be that when the neutron source is placed further into the LSDS, neutrons that are emitted away from the fuel have a greater chance of backscattering off the lead and have less chance of escaping the LSDS. 


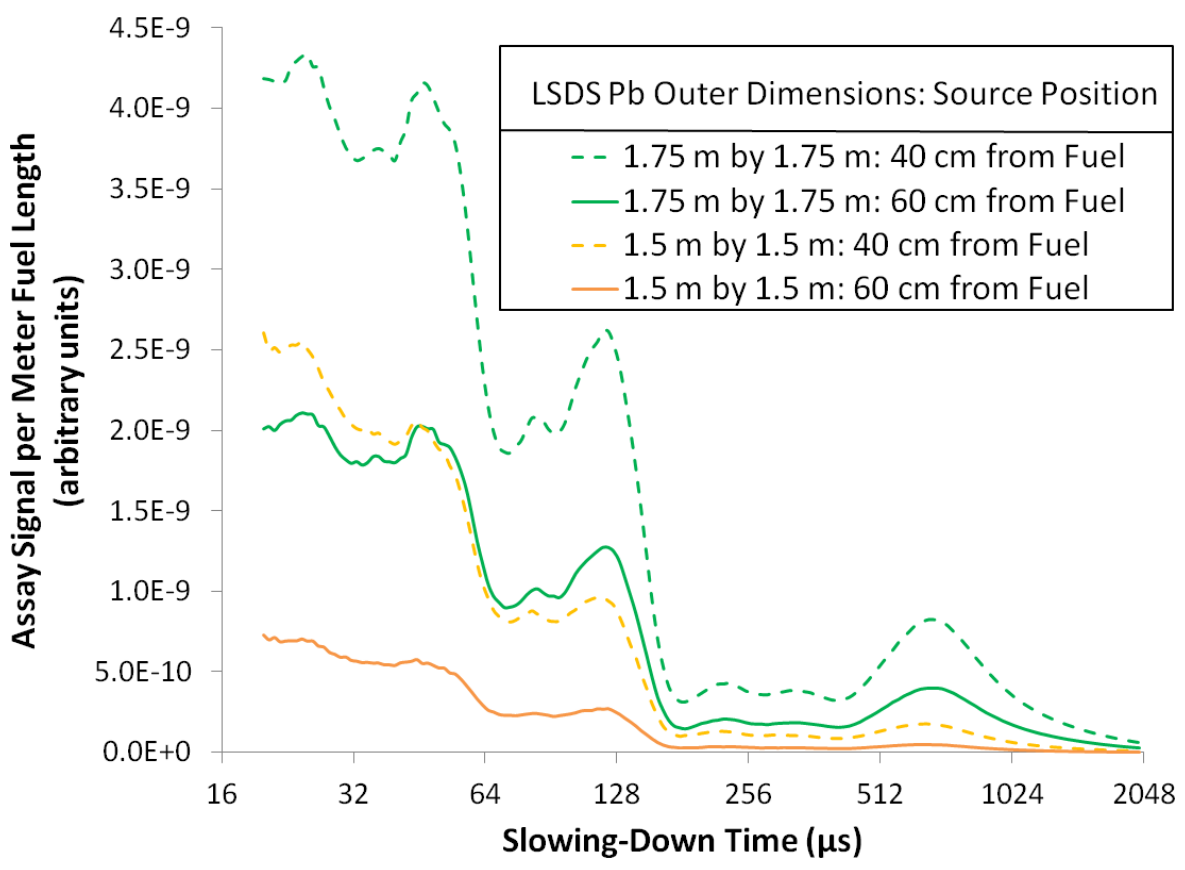

Figure 6-3: Simulated ${ }^{238} \mathrm{U}$ fission chamber signal for cylindrical LSDS instruments of various size (lead outer dimensions). The source distance from the fuel was varied between 40 and $60 \mathrm{~cm}$.

\subsection{Level of ${ }^{235} \mathrm{U}$ impurities and cadmium}

One of the challenges in the LSDS design has been the development of a fast neutron detector. The ideal detector would be sensitive only fast neutrons. A pure ${ }^{238} \mathrm{U}$ fission chamber would have very little sensitivity to neutrons below $0.5 \mathrm{MeV}$, however a pure ${ }^{238} \mathrm{U}$ chamber is not practical, given that even minuscule levels of natural uranium or depleted uranium would introduce significant ${ }^{235} \mathrm{U}$ impurities. It is important to determine what level of ${ }^{235} \mathrm{U}$ impurities is acceptable.

A first qualitative attempt to answer that question has been completed and is shown in the top of Figure 6-4. The assay signals of a ${ }^{238} \mathrm{U}$ fission chamber with various levels of impurities of ${ }^{235} \mathrm{U}$ are shown. No impurities and $4 \mathrm{ppm}{ }^{235} \mathrm{U}$ produce essentially the same signal. While the higher levels of impurities do change the response, they do not dramatically alter the shape of the response. As a result, it may be possible that significantly higher levels of ${ }^{235} \mathrm{U}$ impurities than $4 \mathrm{ppm}$ may be tolerable and provide reasonable uncertainties once applied across the NGSI 64 library.

A follow-up issue to the level of ${ }^{235} \mathrm{U}$ impurities is the possibility of using cadmium-lined fission chambers to reduce the thermal neutron flux in the fission chambers, thus making them less sensitive to ${ }^{235} \mathrm{U}$ impurities. A plot similar to the top of Figure 6-4, but with $1 \mathrm{~mm}$ of cadmium lining around the fission chambers, is shown at the bottom of this figure. The cadmium only modestly reduces the sensitivity to the level of ${ }^{235} \mathrm{U}$ impurities, and it also significantly impacts the shape of the signal. This change in the shape can be more easily seen in Figure 6-5, which also includes the assay signal from a ${ }^{232} \mathrm{Th}$ fission chamber. 


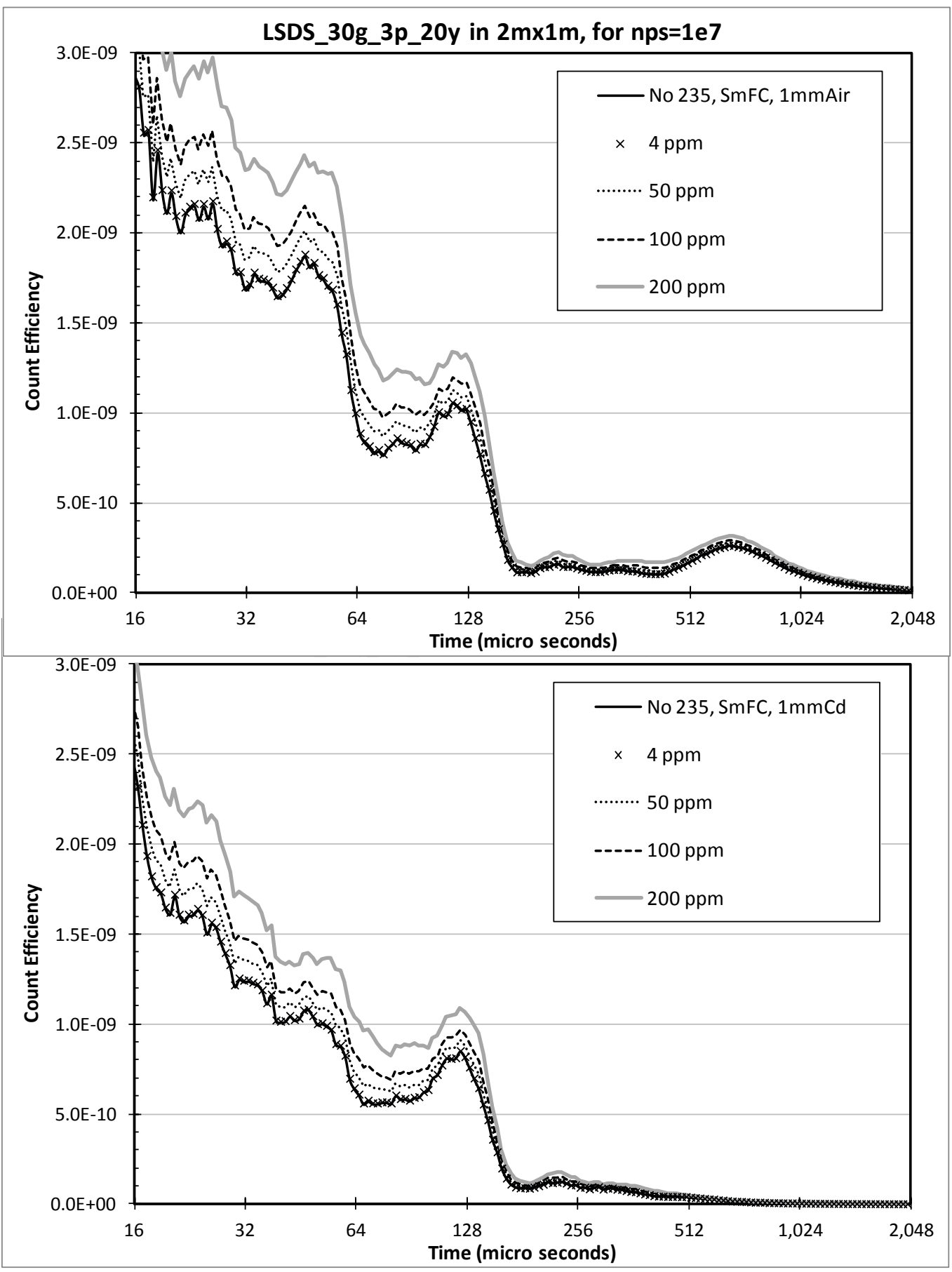

Figure 6-4: Sensitivity to ${ }^{235} \mathrm{U}$ impurities in a ${ }^{238} \mathrm{U}$ fission chamber. The top figure is the count efficiency of the nominal ${ }^{238} \mathrm{U}$ fission chamber with given levels of impurities. The bottom figure is similar, except that the fission chamber has been surrounded by $1 \mathrm{~mm}$ of cadmium. The "SmFC" denotes a certain fission chamber geometry used in the simulations. 


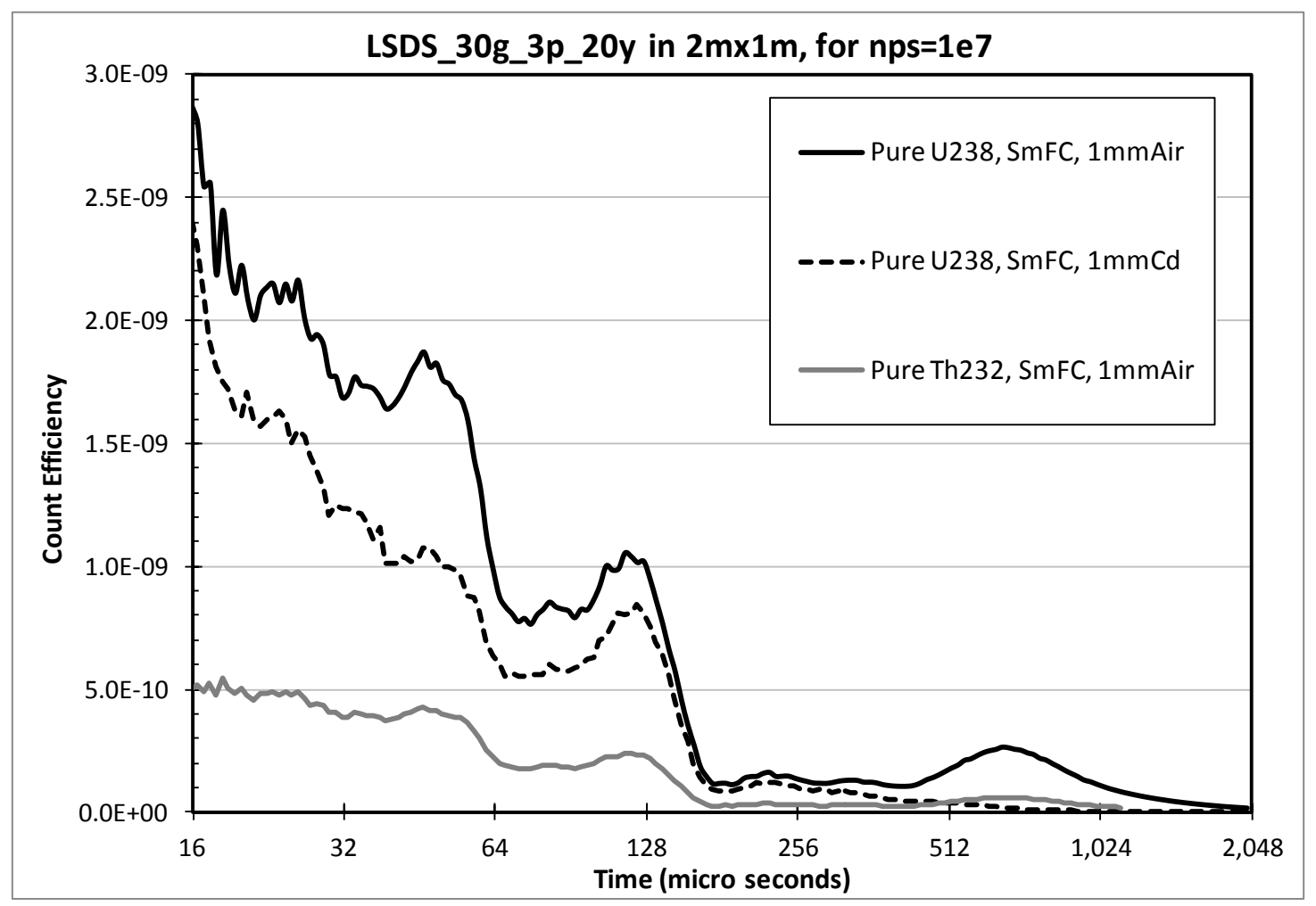

Figure 6-4: Comparison of assay signal response for a pure ${ }^{238} \mathrm{U}$ chamber, a pure ${ }^{232} \mathrm{Th}$ chamber and a ${ }^{238} \mathrm{U}$ chamber lined with $1 \mathrm{~mm}$ of cadmium.

Two general conclusions can be drawn from these studies:

- It is possible that considerably higher than $4 \mathrm{ppm}{ }^{235} \mathrm{U}$ impurities may be tolerable in a ${ }^{238} \mathrm{U}$ fission chamber, and

- Cadmium is not very beneficial. It does not significantly reduce the sensitivity to the level of ${ }^{235} U$ impurities and it significantly alters the shape of the response, possibly destroying some of the signal. 


\subsection{Future Work}

In FY2013, PNNL will continue efforts to develop and refine design requirements of an LSDS for the ultimate purpose of assaying used-fuel assemblies. The requirements include quantitative analysis to justify lead size, placement of neutron source, intensity of neutron source, and the number and type of detectors required. In addition, several steps need to be taken to improve the baseline simulation geometry. These improvements include incorporation of lead impurities, implementation of an electronbased neutron source, and improvement of statistical uncertainties in the simulations. Future efforts will be directed toward more extensive experimental benchmarking of currently implemented time-spectra analysis algorithms. 


\subsection{Conclusions}

The potential for LSDS to provide direct, independent, and accurate assay of uranium and plutonium isotopes in used fuel relies heavily on developing time-spectra analysis methods that can account for the nonlinear effects of self-shielding and neutron absorption by non-fissile isotopes. The time-spectra analysis method development efforts from FY2012 were evaluated using a simulated library of PWR used-fuel assemblies that spans a wide range of initial enrichment, burnup and cooling time (and included pin-to-pin and within-pin burnup variation). The major conclusions of the FY2012 evaluation of the time spectral analysis algorithm are summarized below:

- The calibration-based SVD algorithm demonstrated the ability of the LSDS to perform direct and independent assay of ${ }^{239} \mathrm{Pu}$ and ${ }^{241} \mathrm{Pu}$ to an average uncertainty less than $2 \%$ for the wide parameter space spanned by the NGSI 64 and 27 diversion used-fuel assemblies.

- A calibration set of six fuels provided reasonable uncertainties in the masses.

- Two new time-spectra analysis algorithms were developed in FY2012 that do not rely on plutonium isotopic fission chambers.

- The time-spectra analysis algorithms developed and refined in FY2012 were successfully applied to experimental data measured by RPI with their LSDS in FY2011. Reasonable estimates (RMSE $<5 \%$ ) on ${ }^{235} \mathrm{U}$ mass were obtained for all time-spectra analysis algorithms. More variety in the plutonium experimental data is required for better evaluation of the algorithms.

- The sensitivity of the semi-empirical algorithm to the uncertainties of the calibration masses was determined. For example, $2 \%$ uncertainty in the ${ }^{235} \mathrm{U},{ }^{239} \mathrm{Pu}$, and ${ }^{241} \mathrm{Pu}$ masses in the calibration set fuel assemblies resulted in average RMSE values for ${ }^{239} \mathrm{Pu}+{ }^{241} \mathrm{Pu}$ mass still within $3 \%$.

- The assay signal of the LSDS is sensitive to the center of the assembly at $\sim 55 \%$ compared to the outer edges of the assembly. This may be potentially remedied with the use of small in-core fission chambers inserted into the guide tubes.

- Adequate sensitivity can be achieved with 30-40 metric tons of lead. More lead may improve sensitivity of the approach.

Provided the neutron emission from the interrogation neutron source is fairly isotropic, the neutron source should be located near the center of the LSDS. 


\subsection{References}

[1] N. Peter, "A Shipper-Receiver-Difference Approach for Reprocessing Plants," 8th International Conference on Facility Operations - Safeguards Interface, Portland OR, 2008.

[2] Y. Danon, et al, "Measurements with the high flux lead slowing-down spectrometer at LANL," Nuclear Instruments and Methods in Physics Research B, 261/1-2, pp. 953-955, 2007.

[3] C. Romano, et al, "Measurements of $(\mathrm{n}, \alpha)$ cross-section of small samples using a lead-slowing-downspectrometer," Nuclear Instruments and Methods in Physics Research A, 562/2, pp. 771-773, 2006.

[4] L.E. Smith and N.M. Abdurrahman, "Neutron Spectrometry for the Assay of High Fissile Content Spent Fuel," Nucl. Tech. 140, 328 (2002).

[5] J.J. Ressler, L.E. Smith, and K .K. Anderson, "Lead Slowing Down Spectroscopy for Direct Plutonium Mass Measurements." in 8th International Conference on Facility Operations - Safeguards Interface, Portland OR (2008).

[6] L.E. Smith, KK Anderson, J.J. Ressler, S.D. Kiff, M.W. Shaver, "Time-Spectral Analysis Algorithms for Lead Slowing-Down Spectroscopy of Spent Fuel,” 2009 Institute of Nuclear Materials Management Conference, Tucson, AZ, July 2009.

[7] L.E. Smith, C.J. Gesh, K.K. Anderson, A.M.Casella, and M.W. Shaver, "Lead Slowing-Down Spectroscopy for Direct Measurement of Plutonium in Spent Fuel: NGSI Phase I Report. PNNL20158, Pacific Northwest National Laboratory, Richland, WA. 2010.

[8] S.J. Tobin, et al., "Determination of Plutonium Content in Spent Fuel with Nondestructive Assay," 2009 Institute of Nuclear Materials Management Conference, Tucson, AZ, 2009.

[9] D.B. Pelowitz, "MCNPX User's Manual”, Vol. LA-CP-07-1473 Version 2.60, Department of Energy, Los Alamos National Laboratory, Los Alamos, NM: U.S., 2008.

[10] J.A. Kulisek, et al, "Progress on Establishing the Feasibility of Lead Slowing Down Spectroscopy for Direct Measurement of Plutonium in Used Fuel," 2011 Institute of Nuclear Materials Management Conference, Palm Desert, CA, July 2011.

[11] Y.D. Lee, et al. "Design of a Spent-Fuel Assay Device Using a Lead Spectrometer," Nucl. Sci. Eng. 131/1, pp. 45-61, 1999.

[12] G. H. Golub and C. Reinsch, "Singular Values and Least Squares Solutions," Numer. Math. 14, pp. 403-420, 1970.

[13] J. Kulisek, K. Anderson, A. Casella, C. Gesh, and G. Warren, “Assaying Used Nuclear Fuel Assemblies Using Lead Slowing-Down Spectroscopy and Singular Value Decomposition," submitted to IEEE Trans. Nucl. Sci. and presentation at the SORMA West Conference, Oakland, CA, 2012. 
[14] N. M. Abdurrahman, et Al, "Spent-Fuel Assay Performance and Monte Carlo Analysis of the Rensselaer Slowing-Down-Time Spectrometer," Nuclear Science and Engineering 115, pp. 279-296. (1993).

[15] Dr. Ir. Alessandro Borella (SCK-CEN), personal communication, July 2012.

[16] J. Kulisek, K. Anderson, S. Bowyer, A.M. Casella, C. Gesh, and G. Warren, "Lead Slowing-Down Spectrometry Time Spectral Analysis for Spent Fuel Assay: FY11 Status Report.” PNNL-20769, Pacific Northwest National Laboratory, Richland, WA. 2011.

[17] D. B. Pelowitz, "MCNPX User's Manual”, Vol. LA-CP-11-00438 Version 2.70, Department of Energy, Los Alamos National Laboratory, Los Alamos, NM: U.S., April 2011.

[18] C. Romano, Y. Danon, and D. Beller, "Fuel Assembly Self Shielding of Interrogation Neutrons In a Lead Slowing-Down Spectrometer," $6^{\text {th }}$ American Nuclear Society International Topical Meeting on Nuclear Plant Instrumentation, Control, and Human-Machine Interface Technologies (NPIC\&HMIT 2009), Knoxville, Tennessee, April 5-9, 2009 [on CD-ROM].

[19] D. S. Cramer, R. E. Slovacek, E. B. Bean, R. G. Luce, "Lead Spectrometer for Non-Destructive Nuclear Fuel Assay,” KAPL-M-7449, pp. 10, Knolls Atomic Power Laboratory, 1976. 


\section{Appendix A: Numerical Methods and Fitting Algorithms}

All of the time-spectral analysis algorithms described in this paper incorporated the Poisson variability that would be expected in real data. Poisson statistical counting uncertainty was simulated into the assay signal, $y(t)$, and the isotopic response functions, $x_{i}(t)$. For the assay signal, Poisson counts were simulated in each time bin, $Y(t)$, with expected number of counts equal to $y(t)^{*} S F y^{*} M^{*} N$, where $S F y$ is a scaling factor equal to 0.0485 to account for the absolute detection efficiency of 32 threshold fission chambers (sizes specified in [7]), each lined with a 1-micron layer of active materials (e.g., ${ }^{232} \mathrm{Th}$ or ${ }^{238} \mathrm{U}$ ), $M$ is the total number of neutrons emitted per pulse, and $N$ is the number of pulses utilized in the simulated assay (e.g., one pulse every 10 milliseconds for a 15 -minute assay would result in approximately $1 \times 10^{5}$ pulses). For the isotopic response functions, the simulated Poisson counts, $X_{i}(t)$ used an expected number of counts equal to $x_{i}(t)^{*} S F x^{*} M^{*} N$, where $S F x$ is a scaling factor equal to 0.0025 to account for the absolute detection efficiency of a single fission chamber lined with an active layer of 1 micron of ${ }^{239} \mathrm{Pu},{ }^{241} \mathrm{Pu}$ or ${ }^{235} \mathrm{U}$, also with the fission chamber size described in [7]. For all of the analysis presented in this report, the $M$ and $N$ values were set at $10^{8}$, resulting in a total number of emitted neutrons of $10^{16}$ for the assay of each assembly, the estimated required number of source neutrons, as described in section 4.0 of this report.

The fitting method used for in this work, the Maximum Likelihood Estimation (MLE), involves minimizing the objective function, $R$, the negative log-likelihood, given by

$$
R=\sum_{j=1}^{n}\left(y^{\prime}\left(t_{j}\right)-Y\left(t_{j}\right) \log \left(y^{\prime}\left(t_{j}\right)\right)\right),
$$

where log is the natural logarithm and $y^{\prime}\left(t_{j}\right)$ is the calculated assay signal. For example, y'(ti) for the semi-empirical algorithm is

$$
y^{\prime}\left(t_{j}\right)=f\left(t_{j}\right) C \sum_{i} \frac{m_{i}}{\bar{v}_{i} A_{i}} X_{i}\left(t_{j}\right)
$$

In (A.2), (8) was substituted for $f\left(t_{j}\right)$ when applying the semi-empirical algorithm. 


\section{Appendix B: Use of Isotopically Impure Plutonium Fission Chambers}

\section{B.1 Introduction}

PNNL has developed a semi-empirical algorithms for extracting the isotopic masses of fissile ${ }^{235} \mathrm{U},{ }^{239} \mathrm{Pu}$, and ${ }^{241} \mathrm{Pu}$ present in a used fuel assembly [16]. In the current formulation of the semi-empirical algorithm, the isotopic fission chambers are assumed to each be comprised of $100 \%$ pure isotopic fissile material. It is impractical to assume that $100 \%$ pure isotopic ${ }^{239} \mathrm{Pu}$ or ${ }^{241} \mathrm{Pu}$ fission chambers can be realized for use in an LSDS. Therefore, the objective of this analysis is to determine if the theory upon which the current PNNL LSDS algorithm is still valid if one has only impure isotopic fission chambers available. The theory is first developed, here, for the case of pure isotopic fission chambers. Then, the theory based on the use of impure fission chambers is developed.

\section{B.2 Theory Based on Pure Isotopic Fission Chambers}

The current PNNL algorithms are based on the theory developed in [14]. Using this theory, the assay response, $Y_{238}$, from a pure ${ }^{238} \mathrm{U}$ fission chamber is

$$
Y_{238}(t)=k_{1} \eta_{1}\left(v_{235} N_{235} \sigma_{235}(t) \varphi(t)+v_{239} N_{239} \sigma_{239}(t) \varphi(t)+v_{241} N_{241} \sigma_{241}(t) \varphi(t)\right)
$$

In (B-1), $\eta_{1}$ is the absolute efficiency of the assay detector, $\mathrm{k}_{1}$ is a normalization constant, $N_{i}$ is the number of isotopes of type $i$ present in the fuel assembly, $v$ is the average neutron yield per fission event for isotope $i$, and $\sigma_{i}(t) \varphi(t)$ is the fission reaction for isotope $i$.

The response of a pure isotope fission chamber is given by

$$
X_{i}(t)=k_{2} \eta_{2} n_{i} \sigma_{i}(t) \varphi(t) .
$$

In (B-2), $k_{2}$ and $\eta_{2}$ are the normalization constant and absolute efficiency, respectively, of the isotopic fission chambers. These two quantities are assumed to be the same for each isotopic fission chamber. Also, $n_{i}$ is the number of isotopic nuclei of type $i$ present in the isotopic fission chamber.

Assuming that the shapes of the interrogating neutron flux at the isotopic and assay fission chambers are the same, each of the fission reaction terms, $\sigma_{i}(t) \varphi(t)$, can be eliminated by using (B-2), resulting in

$$
Y_{238}(t)=\frac{k_{1} \eta_{1}}{k_{2} \eta_{2}}\left[v_{235}\left(\frac{N_{235}}{n_{235}}\right) X_{235}(t)+v_{239}\left(\frac{N_{239}}{n_{239}}\right) X_{239}(t)+v_{241}\left(\frac{N_{241}}{n_{241}}\right) X_{241}(t)\right] .
$$

To simplify the analysis, we assume that the number of nuclei present in each of the isotopic fission chambers is unity, and therefore the $n_{i}$ 's drop out of (B-3). This simplification is justified for the purpose of this purely theoretical analysis, since the number of nuclei present in the isotopic fission chambers is a 
constant and independent of the fuel assembly being assayed. Then, factoring out $N_{A}$, Avogadro's number, (B-3) can be written as

$$
Y_{238}(t)=\frac{k_{1} \eta_{1} N_{A}}{k_{2} \eta_{2}}\left[v_{235}\left(\frac{M_{235}}{A_{235}}\right) X_{235}(t)+v_{239}\left(\frac{M_{239}}{A_{239}}\right) X_{239}(t)+v_{241}\left(\frac{M_{241}}{A_{241}}\right) X_{241}(t)\right] \text {. }
$$

We then take in to account the difference in the interrogation neutron flux within the fuel and the isotopic fission chambers, namely $f(t)$, given by

$$
f(t)=\frac{\varphi_{\text {fuel }}(\mathrm{t})}{\varphi_{\text {detectors }}(t)} .
$$

Then, letting $C=\frac{k_{1} \eta_{1} N_{A}}{k_{2} \eta_{2}}$, and multiplying (B-4) by (B-5), we obtain the equation used in the current PNNL time-spectral analysis methods, based on pure isotopic fission chambers:

$$
Y_{238}(t)=f(t) C\left[v_{235}\left(\frac{M_{235}}{A_{235}}\right) X_{235}(t)+v_{239}\left(\frac{M_{239}}{A_{239}}\right) X_{239}(t)+v_{241}\left(\frac{M_{241}}{A_{241}}\right) X_{241}(t)\right] \text {. }
$$

This equation has been demonstrated to be successful in determining the fissile isotopic masses of ${ }^{235} \mathrm{U}$, ${ }^{239} \mathrm{Pu}$, and ${ }^{241} \mathrm{Pu}[16]$.

\section{B.3 Theory Based on Impure (mixed) Plutonium Isotopic Fission Chambers}

In this section, we show how the theory of the previous section can be applied for the case of impure plutonium isotopic fission chambers, with only a slight modification. We assume that a pure ${ }^{235} \mathrm{U}$ isotopic fission chamber is available, such that using (B-2) its response is given by

$$
X_{235}(t)=k_{2} \eta_{2} n_{235} \sigma_{235}(t) \varphi(t) .
$$

We then assume that we have two plutonium fission chambers, one that contains $n_{239}$ atoms of ${ }^{239} \mathrm{Pu}$ and $n_{241}$ atoms of ${ }^{241} \mathrm{Pu}$ while the other contains $\tau_{239}$ atoms of ${ }^{239} \mathrm{Pu}$ and $\tau_{241}$ atoms of ${ }^{241} \mathrm{Pu}$, such that their responses are given by

$$
\begin{aligned}
& X_{P u 1}(t)=k_{2} \eta_{2}\left[n_{239} \sigma_{239}(t) \varphi(t)+n_{241} \sigma_{241}(t) \varphi(t)\right], \\
& X_{P u 2}(t)=k_{2} \eta_{2}\left[\tau_{239} \sigma_{239}(t) \varphi(t)+\tau_{241} \sigma_{241}(t) \varphi(t)\right] .
\end{aligned}
$$

We can then use (B-8) and (B-9) to solve for the fission reaction rates $\sigma_{239}(t) \varphi(t)$ and $\sigma_{241}(t) \varphi(t)$ in terms of the other variables: 


$$
\begin{gathered}
\sigma_{239}(t) \varphi(t)=\left(\frac{1}{k_{2} \eta_{2}}\right)\left[\frac{X_{P u 1}(t)}{n_{239}-n_{241}\left(\frac{\tau_{239}}{\tau_{241}}\right)}-\frac{X_{P u 2}(t)}{\left(\frac{n_{239}}{n_{241}}\right) \tau_{241}-\tau_{239}}\right], \\
\sigma_{241}(t) \varphi(t)=\left(\frac{1}{k_{2} \eta_{2}}\right)\left[\frac{X_{P u 2}(t)}{\tau_{241}-\tau_{239}\left(\frac{n_{241}}{n_{239}}\right)}-\frac{X_{P u 1}(t)}{\left(\frac{\tau_{241}}{\tau_{239}}\right) n_{239}-n_{241}}\right] .
\end{gathered}
$$

In contrast to (4) for the case of pure isotopic fission chambers, by substituting (B-7), (B-10), and (B-11), into (B-1), to obtain

$$
Y_{238}(t)=\frac{k_{1} \eta_{1}}{k_{2} \eta_{2}}\left[v_{235}\left(\frac{N_{235}}{n_{235}}\right) X_{235}(t)+v_{239} N_{239} A(t)+v_{241} N_{241} B(t)\right] .
$$

In (B-12), we have replaced the terms in brackets in (B-10) and (B-11) by $A(t)$ and $B(t)$, respectively. In contrast to (B-6) for the case of assuming pure isotope fission chambers, letting $C=\frac{k_{1} \eta_{1} N_{A}}{k_{2} \eta_{2}}$ and multiplying by $f(t)$ to account for the difference in the flux between the isotopic fission chambers and within the fuel, we obtain

$$
Y_{238}(t)=f(t) C\left[v_{235}\left(\frac{M_{235}}{A_{235}}\right)\left(\frac{1}{n_{235}}\right) X_{235}(t)+v_{239}\left(\frac{M_{239}}{A_{239}}\right) A(t)+v_{241}\left(\frac{M_{241}}{A_{241}}\right) B(t)\right] \text {. }
$$

\section{B.4 MCNPX Simulations for Particular Cases of Pure and Impure Plutonium Isotopic Fission Chambers}

It is shown by (B-13) that the theory developed for the case of pure isotopic fission chambers can be slightly modified for the case of impure plutonium isotopic fission chambers. However, in this section, we give an example in order to provide confidence in this result. For simplicity in the derivation, the number of atoms in each fission chamber is normalized to 1 .

As an example, let us assume that we have a fission chamber containing $80 \mathrm{wt} \%{ }^{239} \mathrm{Pu}$ and $20 \mathrm{wt} \%{ }^{241} \mathrm{Pu}$, such that $n_{239}=0.8013$ and $n_{241}=0.1987$. Let us also assume that we have a fission chamber containing $20 \mathrm{wt} \%{ }^{239} \mathrm{Pu}$ and $80 \mathrm{wt} \%{ }^{241} \mathrm{Pu}$, such that $\tau_{239}=0.7987$ and $\tau_{241}=0.2013$. We will assume that we have a pure isotopic ${ }^{235} \mathrm{U}$ fission chamber, such that $n_{235}=1$. Then, for this particular case,

$$
A(t)=1.3312 X_{P u 1}(t)-0.3312 X_{P u 2}(t) \text { and } B(t)=1.3355 X_{P u 2}(t)-0.3355 X_{P u 1}(t) \text {. }
$$


For this example, using MCNPX, we simulated the assay of a fuel assembly having $30 \mathrm{GWd} / \mathrm{MTU}, 3 \%$ enrichment, and 20 year cooling-time, taken from the LANL 64 used fuel assembly library. In the MCNPX simulation, we specified pure isotopic ${ }^{235} \mathrm{U},{ }^{239} \mathrm{Pu}$, and ${ }^{241} \mathrm{Pu}$ fission chambers as well as the impure plutonium fission chambers having the percent weight compositions mentioned above. This was done using the MCNPX flux multiplier tally, and tallying the expected fission reaction rates in each of the isotopic fission chambers.

The MCNPX simulation was done in order to verify the mathematical correctness of (B-13). From the MCNPX simulation results, for the pure isotopic fission chamber case, we calculated $f(t) C$. This was done by dividing $Y_{238}(t)$ by term in brackets in (B-6), using the known masses of ${ }^{235} \mathrm{U},{ }^{239} \mathrm{Pu}$, and ${ }^{241} \mathrm{Pu}$ in the used fuel assembly. This value of $f(t) C$ was then substituted into (B-13) to calculate $Y_{238}(t)$, along with the responses of the mixed plutonium fission chambers. As shown in Figure B-1, the assay detector response calculated using (B-13) and that tallied directly from MCNPX are identical, verifying the mathematical correctness of (B-13).

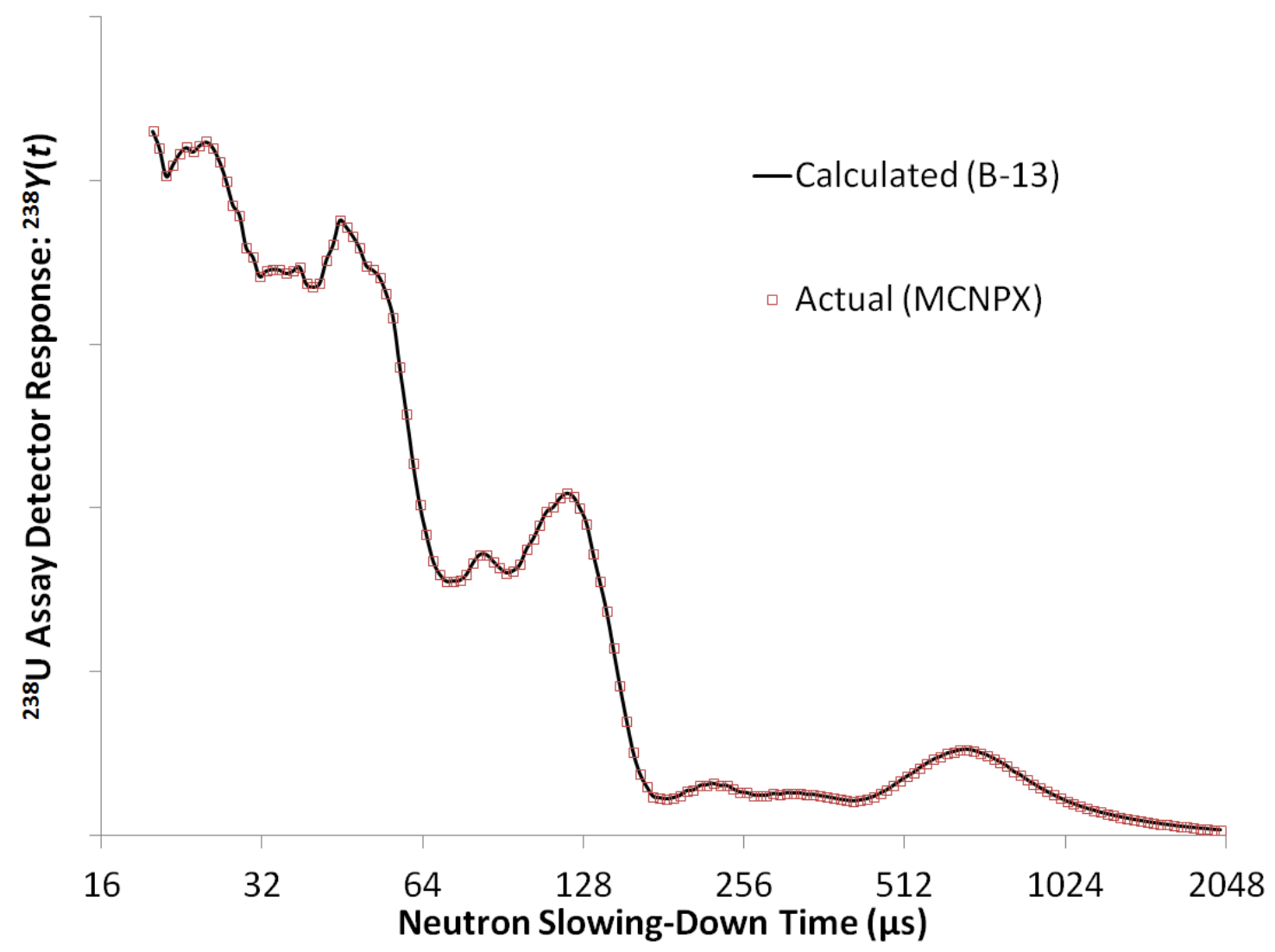

Figure B-1: Calculated and actual MCNPX-tallied ${ }^{238} \mathrm{U}$ assay detector responses 


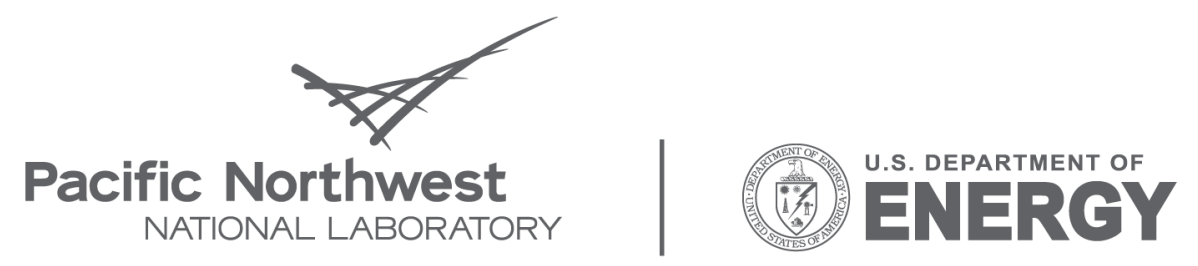

Proudly Operated by Battelle Since 1965

902 Battelle Boulevard

P.O. Box 999

Richland, WA 99352

1-888-375-PNNL (7665)

www.pnl.gov 